TRANSACTIONS OF THE

AMERICAN MATHEMATICAL SOCIETY

Volume 361, Number 6, June 2009, Pages 3125-3157

S 0002-9947(08)04827-7

Article electronically published on December 30, 2008

\title{
ON THE ANALYTICITY OF THE SEMIGROUP GENERATED BY THE STOKES OPERATOR WITH NEUMANN-TYPE BOUNDARY CONDITIONS ON LIPSCHITZ SUBDOMAINS OF RIEMANNIAN MANIFOLDS
}

\author{
MARIUS MITREA AND SYLVIE MONNIAUX
}

\begin{abstract}
We study the analyticity of the semigroup generated by the Stokes operator equipped with Neumann-type boundary conditions on $L^{p}$ spaces in Lipschitz domains. Our strategy is to regularize this operator by considering the Hodge Laplacian, which has the additional property that it commutes with the Leray projection.
\end{abstract}

\section{INTRODUCTION}

This work is concerned with the study of the analyticity of the semigroup associated with the linearized, time-dependent Stokes system with Neumann boundary conditions

$$
\begin{aligned}
& \partial_{t} u-\Delta u+\nabla \pi=f, \quad \operatorname{div} u=0 \text { in } \Omega \times(0, T), \\
& \nu \times\left.\operatorname{curl} u\right|_{\partial \Omega \times(0, T)}=0,\left.\quad \nu \cdot u\right|_{\partial \Omega \times(0, T)}=0,\left.\quad u\right|_{t=0}=u_{o} \text { in } \Omega,
\end{aligned}
$$

as well as the parabolic Maxwell system equipped with a perfectly conductive wall condition, i.e.,

$$
\begin{aligned}
& \partial_{t} u+\operatorname{curl} \operatorname{curl} u=f, \quad \operatorname{div} u=0 \text { in } \Omega \times(0, T), \\
& \nu \times\left. u\right|_{\partial \Omega \times(0, T)}=0,\left.\quad u\right|_{t=0}=u_{o} \text { in } \Omega .
\end{aligned}
$$

In (1.1), $u$ and $\pi$ stand, respectively, for the velocity field and pressure of a fluid occupying a domain $\Omega$, whereas, in (1.2), $u$ denotes the magnetic field propagating inside of the domain $\Omega$. In both cases, $u_{o}$ denotes the initial datum, $f$ is a given, divergence-free field and $\nu$ stands for the unit outward normal to $\partial \Omega$. For a discussion of the relationship between the Neumann (or, as they are occasionally called, "free-boundary") conditions in (1.1) and the more traditionally used Navier's slip boundary conditions to the effect that

$$
\left\{\begin{array}{rlll}
\nu \cdot u & =0 & \text { on } & (0, T) \times \partial \Omega \\
{\left[\left(\nabla u+\nabla u^{\top}\right) \nu\right]_{\text {tan }}} & =0 & \text { on } & (0, T) \times \partial \Omega,
\end{array}\right.
$$

the interested reader is referred to [21].

Received by the editors June 25, 2007.

2000 Mathematics Subject Classification. Primary 42B30, 46A16; Secondary 46E35, 35J25.

Key words and phrases. Hodge-Laplacian, Lipschitz domains, analytic semigroup.

The first author was supported by the NSF grants DMS - 0400639 and DMS FRG - 0456306.

The second author was supported by a UMC Miller Scholarship grant.

(C)2008 American Mathematical Society 3125

Reverts to public domain 28 years from publication 
Systems such as (1.1) and (1.2) naturally arise in the process of linearizing some basic nonlinear evolution problems in mathematical physics, such as the NavierStokes equations and certain problems related to the Ginzburg-Landau model for superconductivity and magneto-hydrodynamics. A more detailed discussion in this regard can be found in the monographs [7] by T.G. Cowling, 16] by L.D. Landau and E.M. Lifshitz, 25] by M.E. Taylor and [8] by R. Dautray and J.-L. Lions. The interested reader may also consult the papers [4, 3] by T. Akiyama, H. Kasai, Y. Shibata and M. Tsutsumi, [5] by V. Barbu, T. Havarneanu, C. Popa and S.S. Sritharan, 10] by E.B. Fabes, J.E. Lewis and N.M. Rivière, 24 by M.Sermange and R. Temam, and 27] by N. Yamaguchi, as well as the literature cited therein.

In a suitable $L^{2}$ context, the stationary versions of (1.1) and (1.2) have unique (finite energy) weak solutions. This is most elegantly seen using the so-called $\{\mathcal{H}, \mathcal{V}, a\}$ formalism as in [8] which, among other things, also gives that the associated solution operators generate analytic semigroups in (appropriate subspaces of) $L^{2}$. Thus, the natural issue which arises here is whether the same is true in the $L^{p}$ context, with $p \neq 2$. This aspect, which is particularly relevant when dealing with nonlinear versions of (1.1)-(1.2), is intimately connected with resolvent estimates for the stationary versions of (1.1)-(1.2). More specifically, for $\lambda \in \mathbb{C}$ and $1<p<\infty$, consider the boundary-value problems

$$
\begin{aligned}
& \lambda u-\Delta u+\nabla \pi=f \in L^{p}(\Omega)^{3} \text { with } \operatorname{div} f=0,\left.\quad \nu \cdot f\right|_{\partial \Omega}=0, \\
& \operatorname{div} u=0 \text { in } \Omega, \\
& u, \operatorname{curl} u \in L^{p}(\Omega)^{3}, \pi \in W^{1, p}(\Omega), \\
& \nu \times\left.\operatorname{curl} u\right|_{\partial \Omega}=0,\left.\quad \nu \cdot u\right|_{\partial \Omega}=0,
\end{aligned}
$$

and

$$
\begin{aligned}
& \lambda u-\operatorname{curl} \operatorname{curl} u=f \in L^{p}(\Omega)^{3}, \quad \operatorname{div} u=0 \text { in } \Omega, \\
& u \in L^{p}(\Omega)^{3}, \operatorname{curl} u \in L^{p}(\Omega)^{3}, \\
& \nu \times\left. u\right|_{\partial \Omega}=0 .
\end{aligned}
$$

In each case, the resolvent estimates alluded to before read

$$
|\lambda|\|u\|_{L^{p}(\Omega)^{3}} \leq C(\Omega, p)\|f\|_{L^{p}(\Omega)^{3}},
$$

uniformly in $\lambda$ satisfying $|\arg (\lambda)|<\theta$ for some $\theta>0$.

When the domain $\Omega$ has a sufficiently smooth boundary, such estimates are well-understood. The classical approach utilizes the fact that the boundary-value problems (1.4)-(1.5) are regular elliptic (cf., e.g., [25]) and the so-called "Agmon trick" (cf. [1]). See, for example, 17] where it is shown that (1.6) holds for each $p \in(1, \infty)$ if $\partial \Omega \in C^{\infty}$.

The nature of the problem at hand changes dramatically as $\partial \Omega$ becomes less regular. To illustrate this point, let us recall the following negative result from 9. For each $p>3$ there exists a bounded cone-like domain $\Omega \subset \mathbb{R}^{3}$ for which the resolvent estimate (1.6) fails in the case of the Stokes system equipped with a 
Dirichlet boundary condition, i.e. for

$$
\begin{aligned}
& \lambda u-\Delta u+\nabla \pi=f \in L^{p}(\Omega)^{3} \text { with } \operatorname{div} f=0,\left.\quad \nu \cdot f\right|_{\partial \Omega}=0, \\
& \operatorname{div} u=0 \text { in } \Omega,\left.\quad u\right|_{\partial \Omega}=0, \\
& u \in W^{1, p}(\Omega)^{3}, \pi \in L^{p}(\Omega) .
\end{aligned}
$$

The sharp nature of the aforementioned counterexample is also underscored by the following intriguing conjecture made by M.E. Taylor in [26].

Taylor's conjecture. For a given bounded Lipschitz domain $\Omega \subset \mathbb{R}^{3}$ there exists $\varepsilon=\varepsilon(\Omega)>0$ such that the Stokes operator associated with (1.7) generates an analytic semigroup on $L^{p}$ provided $3 / 2-\varepsilon<p<3+\varepsilon$.

The range of $p$ 's in the above conjecture is naturally dictated by the mapping properties of the Leray projection

$$
P_{p}: L^{p}(\Omega)^{3} \longrightarrow\left\{u \in L^{p}(\Omega)^{3}: \operatorname{div} u=0, \nu \cdot u=0\right\} .
$$

When $p=2$ this is taken to be the canonical orthogonal projection and is obviously bounded, but the issue of whether this extends to a bounded operator in the context of (1.8) for other values of $p$ is considerably more subtle. Indeed, it has been shown in [11] that for any bounded Lipschitz domain in $\mathbb{R}^{n}$ the operator (1.8) is bounded precisely for $3 / 2-\varepsilon<p<3+\varepsilon$ for some $\varepsilon=\varepsilon(\Omega)>0$ and that this result is sharp in the class of Lipschitz domains.

That any $p \in(1, \infty)$ will do in the case when the domain $\Omega$ has a smooth boundary has been proved by Y. Giga in his seminal paper 12. The resolvent estimates established there also allowed the author to describe the domains of the fractional powers of the Stokes operator.

In this paper, we are able to prove the analogue of Taylor's conjecture for the Stokes system equipped with Neumann-type boundary conditions. Our approach makes essential use of the recent progress in understanding Poisson-type problems for the Hodge Laplacian such as

$$
\begin{aligned}
& -\Delta u=f \in L^{p}(\Omega)^{3}, \\
& \left.\nu \cdot u\right|_{\partial \Omega}=0, \quad \nu \times\left.\operatorname{curl} u\right|_{\partial \Omega}=0, \\
& u \in L^{p}(\Omega)^{3}, \operatorname{div} u \in W^{1, p}(\Omega), \operatorname{curl} u \in L^{p}(\Omega)^{3} .
\end{aligned}
$$

Recently, it has been shown in [20] that, given a three-dimensional Lipschitz domain, there exist

$$
1 \leq p_{\Omega}<\frac{3}{2}<3<q_{\Omega} \leq \infty, \quad 1 / p_{\Omega}+1 / q_{\Omega}=1,
$$

such that (1.9) is well-posed if and only if $p \in\left(p_{\Omega}, q_{\Omega}\right)$. Here, the index $p_{\Omega}$ can be further defined in terms of the critical exponents intervening in the (regular) Dirichlet and Neumann problems for the Laplace-Beltrami operator in $\Omega$ (as well as its complement), when optimal $L^{p}$ estimates for the associated nontangential maximal function are sought. One feature of $\Omega$ which influences the size of $p_{\Omega}$ is the local oscillations of the unit conormal $\nu$ to $\partial \Omega$. In particular, $p_{\Omega}=1$ (and, hence, $q_{\Omega}=\infty$ ) when $\nu$ belongs to the Sarason class of functions of vanishing mean oscillations (which is the case if, e.g., $\partial \Omega \in C^{1}$ ). Furthermore, for a Lipschitz polyhedron in the Euclidean setting, $p_{\Omega}$ can be estimated in terms of the dihedral angles involved. 
Similar results have been proved earlier in [19] for dimensions greater than three, albeit for the range $p \in\left(p_{\Omega}, q_{\Omega}\right)$ with

$$
1 \leq p_{\Omega}<2<q_{\Omega} \leq \infty, \quad 1 / p_{\Omega}+1 / q_{\Omega}=1 .
$$

Here, one should typically regard the indices $p_{\Omega}, q_{\Omega}$ as small perturbations of 2 .

One of our key results states that the Hodge Laplacian, in the context of (1.9), generates an analytic semigroup in $L^{p}$ for any $p \in\left(p_{\Omega}, q_{\Omega}\right)$. From this and the fact that the Leray projection commutes with the semigroup generated by the Hodge Laplacian, it is then possible to prove a similar conclusion for the Stokes and Maxwell operators acting on $L^{p}$ (cf. Theorem 7.3 and Theorem 7.4 for precise statements). Thus, from this perspective, one key feature of our approach is to embed the Stokes (and Maxwell) system into a more general (and, ultimately, more manageable) elliptic problem, in a way which allows us to return to the original system by specializing the type of data allowed in the formulation of the problem.

We carry out this program in the context of differential forms on Lipschitz subdomains of a smooth, compact, boundaryless Riemannian manifold $\mathcal{M}$. This is both notationally convenient and natural from a geometric point of view. It also allows for a more general setting than previously considered in the literature even in the case when $\partial \Omega \in C^{1}$ (semigroup methods for differential forms in the smooth context are discussed in, e.g., P.E. Conner's book 6]). Roughly speaking, at the level of vector fields (identified with 1-differential forms), our main result reads as follows (see $\S 7$ for a precise statement).

Theorem. Let $\Omega \subset \mathcal{M}$ be a Lipschitz domain, $1<p<\infty$, and let $A_{p}$ denote the Stokes operator, i.e. the mapping $u \mapsto-\Delta u$, for each vector field $u \in L^{p}\left(\Omega, \Lambda^{1}\right)$ satisfying

$$
\operatorname{div} u=0 \quad \text { in } \Omega, \quad \operatorname{curl} u \in L^{p}\left(\Omega, \Lambda^{1}\right), \quad \nu \times\left.\operatorname{curl} u\right|_{\partial \Omega}=0,\left.\quad \nu \cdot u\right|_{\partial \Omega}=0
$$

and for which there exists a scalar function $\pi \in W^{1, p}(\Omega)$ such that

$$
f:=-\Delta u+\nabla \pi \in L^{p}\left(\Omega, \Lambda^{1}\right) \text { and } \operatorname{div} f=0,\left.\quad \nu \cdot f\right|_{\partial \Omega}=0 .
$$

Then the operator $-A_{p}$ generates an analytic semigroup $\left(e^{-t A_{p}}\right)_{t>0}$ on the space $\left\{f \in L^{p}\left(\Omega, \Lambda^{1}\right): \operatorname{div} f=0,\left.\nu \cdot f\right|_{\partial \Omega}=0\right\}$ whenever $p \in\left(p_{\Omega}, q_{\Omega}\right)$.

Several remarks are in order here. First, it is remarkable that the range of $p$ 's for which the above result is valid agrees with the range predicted by Taylor's conjecture. Second, the case $p=2$ follows directly from standard functional analysis. Hence, from this perspective, our result can be interpreted as stating that the semigroup generated by the Stokes operator on $L^{2}$ extends to an analytic semigroup in $L^{p}$, and the infinitesimal generator of this extension is also identified in the most desirable fashion. Third, the above theorem is most relevant in the treatment of the Navier-Stokes initial boundary value problem

$$
\begin{aligned}
& \partial_{t} u-\Delta u+(u \cdot \nabla) u+\nabla \pi=f, \quad \operatorname{div} u=0 \quad \text { in } \Omega \times(0, T), \\
& \nu \times\left.\operatorname{curl} u\right|_{\partial \Omega \times(0, T)}=0,\left.\quad \nu \cdot u\right|_{\partial \Omega \times(0, T)}=0,\left.\quad u\right|_{t=0}=u_{o} \text { in } \Omega,
\end{aligned}
$$

by viewing it as an abstract Cauchy (evolution) problem; cf., e.g., [8], 23], 25]. It is in this context that the ability to allow $p \neq 2$ plays a particularly significant role. This line of work has recently been pursued in [21]. Finally, we remark that similar 
results are valid in connection with the parabolic Maxwell system (1.2), as well as certain nonlinear versions of it.

One distinctive aspect of our work is that, given the low regularity assumptions we make on the underlying domain $\Omega$, we are forced to work with certain nonstandard Sobolev-type spaces, which are well-adapted to the differential operators at hand (such as the exterior derivative operator $d$ and its formal adjoint $\delta$ ). In particular, issues such as boundary traces and embeddings become more delicate than in the standard theory. In this regard, a key ingredient in our approach is an estimate established in [18, to the effect that

$$
\|u\|_{B_{1 / p}^{p, p \#}\left(\Omega, \Lambda^{\ell}\right)} \leq C(p, \Omega)\left(\|u\|_{L^{p}\left(\Omega, \Lambda^{\ell}\right)}+\|d u\|_{L^{p}\left(\Omega, \Lambda^{\ell+1}\right)}+\|\delta u\|_{L^{p}\left(\Omega, \Lambda^{\ell-1}\right)}\right)
$$

is valid for any Lipschitz subdomain $\Omega$ of $\mathcal{M}$, any $p \in\left(p_{\Omega}, q_{\Omega}\right)$ and any differential form of degree $\ell \in\{0,1, \ldots, n\}, n:=\operatorname{dim} \mathcal{M}$, for which the right-hand side of (1.15) is finite and for which $\nu \wedge u=0$ or $\nu \vee u=0$ on $\partial \Omega$. Also, $d$ stands for the exterior derivative operator, $\delta$ is the formal adjoint of $d, B_{s}^{p, q}, 1<p, q<\infty, s \in \mathbb{R}$, stands for the Besov scale, and $p^{\#}:=\max \{p, 2\}$. While the smoothness exponent $s=1 / p$ obviously improves to $s=1$ in the limiting cases $\ell=0$ and $\ell=n$, the above estimate is, generally speaking, sharp in the class of Lipschitz domains.

Another crucial ingredient in our approach is the solution of the Poisson problem for the Hodge Laplacian, i.e. $\Delta=-\left(d d^{*}+d^{*} d\right)$ in the sense of composition of unbounded operators, with $L^{p}$-data in Lipschitz subdomains of $\mathcal{M}$ from 18 and 20. In particular, this allows us to describe the smoothness properties exhibited by the elements in the domain of $\Delta$, as well as to show that the Riesz transforms $d \delta \Delta^{-1}, \delta d \Delta^{-1}$ extend to bounded operators on $L^{p}\left(\Omega, \Lambda^{\ell}\right)$ whenever $p \in\left(p_{\Omega}, q_{\Omega}\right)$.

The organization of the paper is as follows. In Section 2 we collect basic definitions and preliminary results and introduce most of the notational conventions used throughout this work. The Hodge Laplacian is reviewed in Section 3, along with the Stokes and Maxwell operators. In Section 4 we dwell on issues of regularity for differential forms in the domain of the Hodge Laplacian. Here we record several key results, themselves corollaries of the work in [20] and [19]. Our strategy for determining all $p$ 's for which the Hodge Laplacian generates an analytic semigroup in $L^{p}$ is to start with $p=2$ (when mere functional analysis will do) and then develop a bootstrap-type argument which allows us to incrementally increase the value of the integrability exponent from $p$ to $p^{*}:=n p /(n-1)$. This portion of our analysis involves a delicate inductive scheme which is executed in Section 5. Section 6 is subsequently devoted to proving resolvent estimates for the Hodge Laplacian. Finally, the main results of the paper are stated and proved in Section 7, which deals with the issue of analyticity for the semigroup generated by the Hodge Laplacian, as well as the Stokes and Maxwell operators.

\section{BACKGROUND MATERIAL}

In this section we review a number of basic definitions and collect several known results which are going to be useful for us in the sequel.

2.1. Geometrical preliminaries. Let $\mathcal{M}$ be a smooth, compact, oriented manifold of real dimension $n$, equipped with a smooth metric tensor, $g=\sum_{j, k} g_{j k} d x_{j} \otimes$ $d x_{k}$. Denote by $T \mathcal{M}$ and $T^{*} \mathcal{M}$ the tangent and cotangent bundles to $\mathcal{M}$, respectively. Occasionally, we shall identify $T^{*} \mathcal{M} \equiv T \mathcal{M}$ canonically, via the metric. 
Set $\Lambda^{\ell} T \mathcal{M}$ for the $\ell$-th exterior power of $T \mathcal{M}$. Sections in this vector bundle are $\ell$-differential forms. The Hermitian structure on $T \mathcal{M}$ extends naturally to $T^{*} \mathcal{M}:=\Lambda^{1} T \mathcal{M}$ and, further, to $\Lambda^{\ell} T \mathcal{M}$. We denote by $\langle\cdot, \cdot\rangle$ the corresponding (pointwise) inner product. The volume form on $\mathcal{M}$, denoted in the sequel by $\omega$, is the unique unitary, positively oriented differential form of maximal degree on $\mathcal{M}$. In local coordinates, $\omega:=\left[\operatorname{det}\left(g_{j k}\right)\right]^{1 / 2} d x_{1} \wedge d x_{2} \wedge \ldots \wedge d x_{n}$. In the sequel, we denote by $d V$ the Borelian measure induced by $\omega$ on $\mathcal{M}$, i.e., $d V=\left[\operatorname{det}\left(g_{j k}\right)\right]^{1 / 2} d x_{1} d x_{2} \ldots d x_{n}$. Going further, we introduce the Hodge star operator as the unique vector bundle morphism $*: \Lambda^{\ell} T \mathcal{M} \rightarrow \Lambda^{n-\ell} T \mathcal{M}$ such that $u \wedge(* u)=|u|^{2} \omega$ for each $u \in \Lambda^{\ell} T \mathcal{M}$. In particular, $\omega=* 1$ and

$$
u \wedge(* v)=\langle u, v\rangle \omega, \quad \forall u \in \Lambda^{\ell} T \mathcal{M}, \forall v \in \Lambda^{\ell} T \mathcal{M} .
$$

The interior product between a 1 -form $\nu$ and an $\ell$-form $u$ is then defined by

$$
\nu \vee u:=(-1)^{\ell(n+1)} *(\nu \wedge * u)
$$

Let $d$ stand for the (exterior) derivative operator and denote by $\delta$ its formal adjoint (with respect to the metric introduced above). For further reference some basic properties of these objects are summarized below.

Proposition 2.1. For an arbitrary 1 -form $\nu, \ell$-form $u,(n-\ell)$-form $v$, and $(\ell+1)$ form $w$, the following are true:

(1) $\langle u, * v\rangle=(-1)^{\ell(n-\ell)}\langle * u, v\rangle$ and $\langle * u, * v\rangle=\langle u, v\rangle$. Also, $* * u=(-1)^{\ell(n-\ell)} u$;

(2) $\langle\nu \wedge u, w\rangle=\langle u, \nu \vee w\rangle$;

(3) $*(\nu \wedge u)=(-1)^{\ell} \nu \vee(* u)$ and $*(\nu \vee u)=(-1)^{\ell+1} \nu \wedge(* u)$;

(4) $* \delta=(-1)^{\ell} d *, \delta *=(-1)^{\ell+1} * d$, and $\delta=(-1)^{n(\ell+1)+1} * d *$ on $\ell$-forms;

(5) $-(d \delta+\delta d)=\Delta$, the Hodge Laplacian on $\mathcal{M}$.

Let $\Omega$ be a Lipschitz subdomain of $\mathcal{M}$. That is, $\partial \Omega$ can be described in appropriate local coordinates by means of graphs of Lipschitz functions. Then the unit conormal $\nu \in T^{*} \mathcal{M}$ is defined a.e., with respect to the surface measure $d \sigma$, on $\partial \Omega$. For any two sufficiently well-behaved differential forms (of compatible degrees) $u$, $w$ we then have

$$
\int_{\Omega}\langle d u, w\rangle d V=\int_{\Omega}\langle u, \delta w\rangle d V+\int_{\partial \Omega}\langle u, \nu \vee w\rangle d \sigma
$$

2.2. Sobolev and Besov spaces of differential forms. The Sobolev (or potential) class $L_{\alpha}^{p}(\mathcal{M}), 1<p<\infty, \alpha \in \mathbb{R}$, is obtained by lifting the Euclidean scale $L_{\alpha}^{p}\left(\mathbb{R}^{n}\right):=\left\{(I-\Delta)^{-\alpha / 2} f: f \in L^{p}\left(\mathbb{R}^{n}\right)\right\}$ to $\mathcal{M}$ (via a $C^{\infty}$ partition of unity and a pull-back). For a Lipschitz subdomain $\Omega$ of $\mathcal{M}$, we denote by $L_{\alpha}^{p}(\Omega)$ the restriction of elements in $L_{\alpha}^{p}(\mathcal{M})$ to $\Omega$, and set $L_{\alpha}^{p}\left(\Omega, \Lambda^{\ell}\right)=L_{\alpha}^{p}(\Omega) \otimes \Lambda^{\ell} T \mathcal{M}$, i.e. the collection of $\ell$-forms with coefficients in $L_{\alpha}^{p}(\Omega)$. In particular, $L^{p}\left(\Omega, \Lambda^{\ell}\right)$ stands for the space of $\ell$-differential forms with $p$-th power integrable coefficients in $\Omega$.

Let us also note here that if $p, p^{\prime} \in(1, \infty)$ are such that $1 / p+1 / p^{\prime}=1$, then

$$
\left(L_{s}^{p}\left(\Omega, \Lambda^{\ell}\right)\right)^{*}=L_{-s}^{p^{\prime}}\left(\Omega, \Lambda^{\ell}\right), \quad \forall s \in(-1+1 / p, 1 / p) .
$$

The Besov spaces $B_{s}^{p, q}\left(\Omega, \Lambda^{\ell}\right), 1<p, q<\infty, s \in \mathbb{R}$, can be introduced in a similar manner; alternatively, this may be obtained from the Sobolev scale via real interpolation. 
Next, denote by $L_{1}^{p}(\partial \Omega)$ the Sobolev space of functions in $L^{p}(\partial \Omega)$ with tangential gradients in $L^{p}(\partial \Omega), 1<p<\infty$. Besov spaces on $\partial \Omega$ can then be introduced via real interpolation, i.e.

$$
B_{s}^{p, q}(\partial \Omega):=\left(L^{p}(\partial \Omega), L_{1}^{p}(\partial \Omega)\right)_{s, q}, \quad \text { with } 0<s<1,1<p, q<\infty .
$$

Finally, if $1<p, q<\infty$ and $1 / p+1 / p^{\prime}=1,1 / q+1 / q^{\prime}=1$, we define

$$
B_{-s}^{p, q}(\partial \Omega):=\left(B_{s}^{p^{\prime}, q^{\prime}}(\partial \Omega)\right)^{*}, \quad 0<s<1,
$$

and, much as before, set $B_{s}^{p, q}\left(\partial \Omega, \Lambda^{\ell}\right):=B_{s}^{p, q}(\partial \Omega) \otimes \Lambda^{\ell} T \mathcal{M}$.

Recall (cf. [14, 13]) that the trace operator

$$
\operatorname{Tr}: L_{s}^{p}\left(\Omega, \Lambda^{\ell}\right) \longrightarrow B_{s-\frac{1}{p}}^{p, p}\left(\partial \Omega, \Lambda^{\ell}\right)
$$

is well-defined, bounded and onto if $1<p<\infty$ and $\frac{1}{p}<s<1+\frac{1}{p}$. Furthermore, the trace operator has a bounded right inverse

$$
\operatorname{Ex}: B_{s-\frac{1}{p}}^{p, p}\left(\partial \Omega, \Lambda^{\ell}\right) \longrightarrow L_{s}^{p}\left(\Omega, \Lambda^{\ell}\right)
$$

and if $1<p<\infty, \frac{1}{p}<\alpha<1+\frac{1}{p}$, then

$$
\operatorname{Ker}(\operatorname{Tr})=\text { the closure of } C_{o}^{\infty}\left(\Omega, \Lambda^{\ell}\right) \text { in } L_{s}^{p}\left(\Omega, \Lambda^{\ell}\right) \text {. }
$$

For $1<p<\infty, s \in \mathbb{R}$, and $\ell \in\{0,1, \ldots, n\}$ we next introduce

$$
\begin{aligned}
& \mathcal{D}_{\ell}^{p}(\Omega ; d):=\left\{u \in L^{p}\left(\Omega, \Lambda^{\ell}\right): d u \in L^{p}\left(\Omega, \Lambda^{\ell+1}\right\},\right. \\
& \mathcal{D}_{\ell}^{p}(\Omega ; \delta):=\left\{u \in L^{p}\left(\Omega, \Lambda^{\ell}\right): \delta u \in L^{p}\left(\Omega, \Lambda^{\ell-1}\right)\right\},
\end{aligned}
$$

equipped with the natural graph norms. Throughout the paper, all derivatives are taken in the sense of distributions.

Inspired by the identity (2.3), if $1<p<\infty$ and $u \in \mathcal{D}_{\ell}^{p}(\Omega ; \delta)$, we then define $\nu \vee u \in B_{-\frac{1}{p}}^{p, p}\left(\partial \Omega, \Lambda^{\ell-1}\right)$ by

$$
\langle\nu \vee u, \varphi\rangle:=-\langle\delta u, \Phi\rangle+\langle u, d \Phi\rangle
$$

for any $\varphi \in B_{\frac{1}{p}}^{p^{\prime}, p^{\prime}}\left(\partial \Omega, \Lambda^{\ell-1}\right), 1 / p+1 / p^{\prime}=1$, and any $\Phi \in L_{1}^{p^{\prime}}\left(\Omega, \Lambda^{\ell-1}\right)$ with $\operatorname{Tr} \Phi=\varphi$. Note that (2.4), (2.9) imply that the operator

$$
\nu \vee \cdot: \mathcal{D}_{\ell}^{p}(\Omega ; \delta) \longrightarrow B_{-\frac{1}{p}}^{p, p}\left(\partial \Omega, \Lambda^{\ell-1}\right)
$$

is well-defined, linear and bounded for each $p \in(1, \infty)$, i.e.

$$
\|\nu \vee u\|_{B_{-\frac{1}{p}}^{p, p}\left(\partial \Omega, \Lambda^{\ell-1}\right)} \leq C\left(\|u\|_{L_{s}^{p}\left(\Omega, \Lambda^{\ell}\right)}+\|\delta u\|_{L_{s}^{p}\left(\Omega, \Lambda^{\ell-1}\right)}\right) .
$$

The range of the operator (2.13) will be denoted by

$$
\mathcal{X}_{\ell}^{p}(\partial \Omega):=\left\{\nu \vee u: u \in \mathcal{D}_{\ell+1}^{p}(\Omega ; \delta)\right\} \hookrightarrow B_{-\frac{1}{p}}^{p, p}\left(\partial \Omega, \Lambda^{\ell}\right),
$$

which we equip with the natural "infimum" norm. It follows that the operator

$$
\begin{aligned}
& \delta_{\partial}: \mathcal{X}_{\ell}^{p}(\partial \Omega) \longrightarrow \mathcal{X}_{\ell-1}^{p}(\partial \Omega) \\
& \delta_{\partial} f:=-\nu \vee \delta w, \quad \text { if } f=\nu \vee w, \quad w \in \mathcal{D}_{\ell+1}^{p}(\Omega ; \delta)
\end{aligned}
$$

is well-defined, linear and bounded. 
Other spaces of interest for us here are defined as follows. For $1<p<\infty, s \in \mathbb{R}$, and $\ell \in\{0,1, \ldots, n\}$, consider

$$
\mathcal{D}_{\ell}^{p}\left(\Omega ; \delta_{\vee}\right):=\left\{u \in L^{p}\left(\Omega, \Lambda^{\ell}\right): \delta u \in L^{p}\left(\Omega, \Lambda^{\ell-1}\right), \nu \vee u=0\right\},
$$

once again equipped with the natural graph norm.

For further use, we record here a useful variation on the integration by parts formula (2.3), namely that if $1<p, p^{\prime}<\infty$ satisfy $1 / p+1 / p^{\prime}=1$, then

$$
\langle d u, v\rangle=\langle u, \delta v\rangle, \quad \forall u \in \mathcal{D}_{\ell}^{p}(\Omega ; d), \forall v \in \mathcal{D}_{\ell}^{p^{\prime}}\left(\Omega ; \delta_{\vee}\right) .
$$

2.3. The $\{\mathcal{H}, \mathcal{V}, a\}$ formalism. Let $\mathcal{V}$ be a reflexive Banach space continuously and densely embedded into a Hilbert space $\mathcal{H}$ so that, in particular,

$$
\mathcal{V} \hookrightarrow \mathcal{H} \hookrightarrow \mathcal{V}^{*}
$$

and assume that

$$
a(\cdot, \cdot): \mathcal{V} \times \mathcal{V} \longrightarrow \mathbb{C}
$$

is a sesquilinear, bounded form. Then

$$
A_{o}: \mathcal{V} \longrightarrow \mathcal{V}^{*}, \quad A_{o} u:=a(u, \cdot) \in \mathcal{V}^{*}, \quad \forall u \in \mathcal{V},
$$

is a linear, bounded operator satisfying

$$
\mathcal{V}^{*}\left\langle A_{o} u, v\right\rangle_{\mathcal{V}}=a(u, v), \quad \forall u, v \in \mathcal{V} .
$$

Assume further that $a(\cdot, \cdot)$ is symmetric and coercive, in the sense that there exist $C_{1}, C_{2}>0$ such that

$$
\operatorname{Re} a(u, u)+C_{1}\|u\|_{\mathcal{H}}^{2} \geq C_{2}\|u\|_{\mathcal{V}}^{2}, \quad \forall u \in \mathcal{V} .
$$

Then

$$
A_{o}: \mathcal{V} \longrightarrow \mathcal{V}^{*} \quad \text { is bounded and selfadjoint. }
$$

Furthermore, $A_{o}$ is invertible if the constant $C_{1}$ appearing in (2.23) can be taken to be zero. Going further, take $A$ to be the part of $A_{o}$ in $\mathcal{H}$, i.e.

$$
A:=\left.A_{o}\right|_{\operatorname{Dom}(A)}: \mathcal{H} \longrightarrow \mathcal{H}
$$

where

$$
\operatorname{Dom}(A):=\left\{u \in \mathcal{V}: A_{o} u \in \mathcal{H}\right\} .
$$

Hence, (2.25)-(2.26) is an unbounded, selfadjoint operator on $\mathcal{H}$. Furthermore, there exists $\theta \in(0, \pi / 2)$ such that

$$
\left\|(\lambda I-A)^{-1}\right\| \leq \frac{C}{|\lambda|}, \quad \theta<|\arg (\lambda)| \leq \pi,
$$

i.e., $A$ is sectorial; cf., e.g., 8 . In particular, the operator $-A$ generates an analytic semigroup on $\mathcal{H}$ according to the formula

$$
e^{-z A} f:=\frac{1}{2 \pi i} \int_{\Gamma_{\theta^{\prime}}} e^{-\lambda z}(\lambda I-A)^{-1} f d \lambda, \quad|\arg (z)|<\pi / 2-\theta^{\prime},
$$

where $\theta^{\prime} \in(\theta, \pi / 2)$ and $\Gamma_{\theta^{\prime}}:=\left\{r e^{i \theta^{\prime}}: r>0\right\}$; see, e.g., 8], 23, 25]. Finally, when $A$ is invertible (which is the case if we can take $C_{1}=0$ in (2.23)), then the semigroup $\left(e^{-t A}\right)_{t>0}$ is bounded. 
In closing, we would like to point out that the above formalism (discussed in detail in, e.g., 8]) is closely related to K.O. Friedrichs' extension method, as described on p. 325 of [15], and on p. 514 of Vol. I of [25].

\section{The Hodge Laplacian and Related operators}

3.1. The Hodge Laplacian. Recall that the $\ell$-th Betti number of $\Omega$, denoted by $b_{\ell}(\Omega)$, is defined as the dimension of the $\ell$-th singular homology group of $\Omega$, viewed as a topological space, over the reals. It has been proved in [18] that for each Lipschitz domain $\Omega \subset \mathcal{M}$ there exist two conjugate exponents

$$
1 \leq p_{\Omega}<2<q_{\Omega} \leq \infty
$$

such that the space

$$
\mathcal{H}^{p}\left(\Omega, \Lambda^{\ell}\right):=\left\{u \in L^{p}\left(\Omega, \Lambda^{\ell}\right): d u=0, \delta u=0, \nu \vee u=0\right\}
$$

is independent of $p$ if $p \in\left(p_{\Omega}, q_{\Omega}\right)$ and has dimension $b_{\ell}(\Omega)$. We shall occasionally abbreviate $\mathcal{H}\left(\Omega, \Lambda^{\ell}\right):=\mathcal{H}^{2}\left(\Omega, \Lambda^{\ell}\right)$.

Consequently, the orthogonal projection of $L^{2}\left(\Omega, \Lambda^{\ell}\right)$ onto $\mathcal{H}\left(\Omega, \Lambda^{\ell}\right)$ extends canonically to a bounded operator

$$
P_{p}: L^{p}\left(\Omega, \Lambda^{\ell}\right) \longrightarrow \mathcal{H}\left(\Omega, \Lambda^{\ell}\right) \hookrightarrow L^{p}\left(\Omega, \Lambda^{\ell}\right) \text { if } p_{\Omega}<p<q_{\Omega},
$$

which has the property that

$$
P_{p}^{*}=P_{p^{\prime}}, \quad p, p^{\prime} \in\left(p_{\Omega}, q_{\Omega}\right), 1 / p+1 / p^{\prime}=1 .
$$

In order to continue, for each $p \in(1, \infty)$ and $\ell \in\{0,1, \ldots, n\}$ we set

$\mathcal{V}^{p}\left(\Omega, \Lambda^{\ell}\right):=\left\{u \in L^{p}\left(\Omega, \Lambda^{\ell}\right): d u \in L^{p}\left(\Omega, \Lambda^{\ell+1}\right), \delta u \in L^{p}\left(\Omega, \Lambda^{\ell-1}\right), \nu \vee u=0\right\}$

$$
=\mathcal{D}^{p}(\Omega ; d) \cap \mathcal{D}^{p}\left(\Omega ; \delta_{\vee}\right),
$$

once again equipped with the natural graph norm. If $p=2$, we introduce the symmetric, coercive quadratic form

$$
Q_{\ell}(u, v):=\langle d u, d v\rangle+\langle\delta u, \delta v\rangle, \quad u, v \in \mathcal{V}^{2}\left(\Omega, \Lambda^{\ell}\right),
$$

and make the following remark.

Proposition 3.1. If $b_{\ell}(\Omega)=0$, then there exists $C>0$ such that

$$
Q_{\ell}(u, u) \geq C\|u\|_{L^{2}\left(\Omega, \Lambda^{\ell}\right)}^{2}, \quad \forall u \in \mathcal{V}^{2}\left(\Omega, \Lambda^{\ell}\right) .
$$

Proof. We shall rely on the estimate

$$
\|u\|_{L_{1 / 2}^{2}\left(\Omega, \Lambda^{\ell}\right)}^{2} \leq C\left(\|u\|_{L^{2}\left(\Omega, \Lambda^{\ell}\right)}^{2}+Q_{\ell}(u, u)\right), \quad \forall u \in \mathcal{V}^{2}\left(\Omega, \Lambda^{\ell}\right),
$$

which has been established in [18. Reasoning by contradiction, assume that (3.7) fails, so that there exists a sequence $u_{j} \in \mathcal{V}^{2}\left(\Omega, \Lambda^{\ell}\right)$ such that $\left\|u_{j}\right\|_{L^{2}\left(\Omega, \Lambda^{\ell}\right)}=1$ for each $j$ and for which $Q_{\ell}\left(u_{j}, u_{j}\right) \rightarrow 0$ as $j \rightarrow \infty$. By (3.8) and Rellich's selection lemma, there is no loss of generality in assuming that

$$
u_{j} \rightarrow u \text { in } L^{2}\left(\Omega, \Lambda^{\ell}\right) \text { for some } u \text { with }\|u\|_{L^{2}\left(\Omega, \Lambda^{\ell}\right)}=1 .
$$

Now, $Q_{\ell}\left(u_{j}, u_{j}\right) \rightarrow 0$ as $j \rightarrow \infty$ forces $d u_{j} \rightarrow 0$ and $\delta u_{j} \rightarrow 0$ in $L^{2}\left(\Omega, \Lambda^{\ell+1}\right)$ and $L^{2}\left(\Omega, \Lambda^{\ell-1}\right)$, respectively, as $j \rightarrow \infty$ and, hence, $d u=0, \delta u=0$. Furthermore, by the continuity of the operator (2.13), we also have $\nu \vee u=0$. Consequently, $u \in \mathcal{H}\left(\Omega, \Lambda^{\ell}\right)=\{0\}$, given that $b_{\ell}(\Omega)=0$. This contradicts (3.9) and proves the proposition. 
Returning to the mainstream discussion, the $\{\mathcal{H}, \mathcal{V}, a\}$ formalism discussed in $\S 2.3$ applies to the context when $\mathcal{V}:=\mathcal{V}^{2}\left(\Omega, \Lambda^{\ell}\right), \mathcal{H}:=L^{2}\left(\Omega, \Lambda^{\ell}\right)$ and $a(u, v):=$ $Q_{\ell}(u, v)$, and yields an unbounded, selfadjoint operator

$$
B: L^{2}\left(\Omega, \Lambda^{\ell}\right) \longrightarrow L^{2}\left(\Omega, \Lambda^{\ell}\right)
$$

whose domain, $\operatorname{Dom}(B)$, consists of all

$$
u \in \mathcal{V}^{2}\left(\Omega, \Lambda^{\ell}\right) \text { such that there exists } C>0 \text { with the property that }
$$

$$
\left|Q_{\ell}(u, v)\right| \leq C\|v\|_{L^{2}\left(\Omega, \Lambda^{\ell}\right)} \text { for all } v \in \mathcal{V}^{2}\left(\Omega, \Lambda^{\ell}\right)
$$

and for which

$$
\langle B u, v\rangle=Q_{\ell}(u, v), \quad u \in \operatorname{Dom}(B), v \in \mathcal{V}^{2}\left(\Omega, \Lambda^{\ell}\right) .
$$

If we now regard the exterior derivative as an unbounded operator

$$
d_{\ell}: L^{2}\left(\Omega, \Lambda^{\ell}\right) \longrightarrow L^{2}\left(\Omega, \Lambda^{\ell+1}\right)
$$

with domain $\mathcal{D}_{\ell}^{2}(\Omega ; d)$ and natural action,

it is not difficult to check that

$$
B=d_{\ell} d_{\ell}^{*}+d_{\ell}^{*} d_{\ell}
$$

in the sense of composition of unbounded operators. Furthermore, under the assumption that $b_{\ell}(\Omega)=0$, this operator is actually invertible on $L^{2}\left(\Omega, \Lambda^{\ell}\right)$. Cf. also 22 for a related discussion.

The latest description of $B$ has a natural analogue in the $L^{p}$ context. The starting point is the observation that the dual of (3.13) is

$$
\begin{aligned}
& d_{\ell}^{*}: L^{2}\left(\Omega, \Lambda^{\ell+1}\right) \longrightarrow L^{2}\left(\Omega, \Lambda^{\ell}\right) \\
& \operatorname{Dom}\left(d_{\ell}^{*}\right)=\mathcal{D}_{\ell+1}^{2}\left(\Omega ; \delta_{\vee}\right), \quad d_{\ell}^{*} u=\delta u, \forall u \in \mathcal{D}_{\ell+1}^{2}\left(\Omega ; \delta_{\vee}\right) .
\end{aligned}
$$

Thus, if $1<p<\infty$, it is natural to consider the unbounded operator

$$
B_{p}: L^{p}\left(\Omega, \Lambda^{\ell}\right) \longrightarrow L^{p}\left(\Omega, \Lambda^{\ell}\right)
$$

with domain $\operatorname{Dom}\left(B_{p}\right)$ consisting of

$$
u \in \mathcal{D}_{\ell}^{p}(\Omega ; d) \cap \mathcal{D}_{\ell}^{p}\left(\Omega ; \delta_{\vee}\right) \text { with } d u \in \mathcal{D}_{\ell+1}^{p}\left(\Omega ; \delta_{\vee}\right), \delta u \in \mathcal{D}_{\ell+1}^{p}(\Omega ; d)
$$

by setting

$$
B_{p} u:=-\Delta u=(d \delta+\delta d) u, \quad \forall u \in \operatorname{Dom}\left(B_{p}\right) .
$$

Note that since $C_{o}^{\infty}\left(\Omega, \Lambda^{\ell}\right)$ is contained in $\operatorname{Dom}\left(B_{p}\right)$, it follows that $B_{p}$ is densely defined.

Proposition 3.2. Let $\Omega \subset \mathcal{M}$ be a Lipschitz domain. Then for each $p_{\Omega}<p<q_{\Omega}$ there exists a linear, bounded operator

$$
G_{p}: L^{p}\left(\Omega, \Lambda^{\ell}\right) \longrightarrow L^{p}\left(\Omega, \Lambda^{\ell}\right),
$$

such that $\operatorname{Im}\left(G_{p}\right) \subset \operatorname{Dom}\left(B_{p}\right)$,

$$
\begin{aligned}
& \left\|G_{p} u\right\|_{L^{p}\left(\Omega, \Lambda^{\ell}\right)}+\left\|d G_{p} u\right\|_{L^{p}\left(\Omega, \Lambda^{\ell+1}\right)}+\left\|\delta G_{p} u\right\|_{L^{p}\left(\Omega, \Lambda^{\ell-1}\right)} \\
& \quad+\left\|d \delta G_{p} u\right\|_{L^{p}\left(\Omega, \Lambda^{\ell}\right)}+\left\|\delta d G_{p} u\right\|_{L^{p}\left(\Omega, \Lambda^{\ell}\right)} \leq C\|u\|_{L^{p}\left(\Omega, \Lambda^{\ell}\right)}
\end{aligned}
$$

and, in the sense of composition of unbounded operators,

$$
B_{p} G_{p}=G_{p} B_{p}=I-P_{p} \text { on } L^{p}\left(\Omega, \Lambda^{\ell}\right) .
$$


Furthermore,

$$
\left(G_{p}\right)^{*}=G_{p^{\prime}}, \quad p_{\Omega}<p, p^{\prime}<q_{\Omega}, 1 / p+1 / p^{\prime}=1
$$

and (with the subscript $\ell$ used to indicate the dependence on the degree),

$$
\begin{aligned}
& d G_{p, \ell}=G_{p, \ell+1} d \text { on } \mathcal{D}_{\ell}^{p}(\Omega ; d), \\
& \delta G_{p, \ell}=G_{p, \ell-1} \delta \text { on } \mathcal{D}_{\ell}^{p}(\Omega ; \delta) .
\end{aligned}
$$

Such a Green operator has been constructed in [20] when $n=3$ and in [19] in the general case. Let us also note here that (3.21) implies

$$
\operatorname{Ker}\left(B_{p}\right)=\mathcal{H}^{p}\left(\Omega, \Lambda^{\ell}\right) \text { whenever } p_{\Omega}<p<q_{\Omega},
$$

and that, thanks to (2.18),

$$
\operatorname{Im}\left(B_{p}\right) \hookrightarrow \operatorname{Ker}\left(P_{p}\right) \text { for each } p \in\left(p_{\Omega}, q_{\Omega}\right) .
$$

Proposition 3.3. For each Lipschitz subdomain $\Omega$ of $\mathcal{M}$ one has

$$
\left(B_{p}\right)^{*}=B_{p^{\prime}}, \quad p_{\Omega}<p, p^{\prime}<q_{\Omega}, 1 / p+1 / p^{\prime}=1 .
$$

Proof. The inclusion $B_{p^{\prime}} \subset\left(B_{p}\right)^{*}$ is immediate from definitions, so it remains to prove the opposite inclusion. To this end, if $u \in \operatorname{Dom}\left(B_{p}^{*}\right)$, then $u \in L^{p}\left(\Omega, \Lambda^{\ell}\right)$ and there exists $w \in L^{p^{\prime}}\left(\Omega, \Lambda^{\ell}\right)$ such that

$$
\langle w, v\rangle=\left\langle u, B_{p} v\right\rangle, \quad \forall v \in \operatorname{Dom}\left(B_{p}\right) .
$$

Choosing $v:=P_{p} \xi$ with $\xi \in L^{p}\left(\Omega, \Lambda^{\ell}\right)$ arbitrary forces $\left\langle w, P_{p} \xi\right\rangle=0$ and, ultimately, $P_{p^{\prime}} w=0$. Next, pick $v:=G_{p} \eta$ with $\eta \in L^{p}\left(\Omega, \Lambda^{\ell}\right)$ arbitrary and write

$$
\begin{aligned}
\left\langle G_{p^{\prime}} w, \eta\right\rangle & =\left\langle w, G_{p^{\prime}} \eta\right\rangle=\langle w, v\rangle \\
& =\left\langle u, B_{p} v\right\rangle=\left\langle u, B_{p} G_{p} \eta\right\rangle=\left\langle u,\left(I-P_{p}\right) \eta\right\rangle \\
& =\left\langle\left(I-P_{p}\right) u, \eta\right\rangle .
\end{aligned}
$$

Since $\eta$ is arbitrary, this forces $\left(I-P_{p}\right) u=G_{p^{\prime}} w$ and, further, $u=G_{p^{\prime}} w+P_{p^{\prime}} u \in$ $\operatorname{Dom}\left(B_{p^{\prime}}\right)$. In addition, $B_{p^{\prime}} u=B_{p^{\prime}} G_{p^{\prime}} w+B_{p^{\prime}} P_{p^{\prime}} u=\left(I-P_{p^{\prime}}\right) w=w$ which shows that $\left(B_{p}\right)^{*} \subset B_{p^{\prime}}$. This finishes the proof of the proposition.

Going further, we now define the Leray projection operator

$$
\mathbb{P}_{p}:=\delta d G_{p}+P_{p}, \quad p_{\Omega}<p<q_{\Omega},
$$

and introduce the spaces

$$
\begin{aligned}
& X^{p}\left(\Omega, \Lambda^{\ell}\right):=\left\{u \in \mathcal{D}_{\ell}^{p}\left(\Omega ; \delta_{\vee}\right): \delta u=0\right\}, \\
& Y^{p}\left(\Omega, \Lambda^{\ell}\right):=\left\{d u: u \in \mathcal{D}_{\ell-1}^{p}(\Omega ; d)\right\} .
\end{aligned}
$$

As a result of the Hodge decompositions proved in [19], it follows that

$$
L^{p}\left(\Omega, \Lambda^{\ell}\right)=X^{p}\left(\Omega, \Lambda^{\ell}\right) \oplus Y^{p}\left(\Omega, \Lambda^{\ell}\right), \quad p_{\Omega}<p<q_{\Omega} .
$$

For further reference we also note the following.

Lemma 3.4. For each $p_{\Omega}<p, p^{\prime}<q_{\Omega}$ with $1 / p+1 / p^{\prime}=1$, the natural integral pairing on $\Omega$ allows for the identification

$$
\left(X^{p}\left(\Omega, \Lambda^{\ell}\right)\right)^{*} \equiv X^{p^{\prime}}\left(\Omega, \Lambda^{\ell}\right) .
$$


Proof. The goal is to show that the mapping

$$
\Phi: X^{p^{\prime}}\left(\Omega, \Lambda^{\ell}\right) \ni u \mapsto\langle u, \cdot\rangle \in\left(X^{p}\left(\Omega, \Lambda^{\ell}\right)\right)^{*}
$$

is an isomorphism. To see that it is one-to-one, assume that $w \in L^{p}\left(\Omega, \Lambda^{\ell}\right)$ is arbitrary and, using (3.33), decompose $w=w_{1}+w_{2}$ with $w_{1} \in X^{p}\left(\Omega, \Lambda^{\ell}\right)$ and $w_{2} \in Y^{p}\left(\Omega, \Lambda^{\ell}\right)$. Then, if $u \in X^{p^{\prime}}\left(\Omega, \Lambda^{\ell}\right)$ is such that $\Phi(u)=0$, it follows from (2.18) and (3.31)-(3.32) that $\langle u, w\rangle=\left\langle u, w_{1}\right\rangle+\left\langle u, w_{2}\right\rangle=0$. Since $w$ was arbitrary, this forces $u=0$ and, hence, $\Phi$ is one-to-one.

To prove that the mapping (3.35) is onto, let $f \in\left(X^{p}\left(\Omega, \Lambda^{\ell}\right)\right)^{*}$ be arbitrary. Since $X^{p}\left(\Omega, \Lambda^{\ell}\right)$ is a closed subspace of $L^{p}\left(\Omega, \Lambda^{\ell}\right)$, the Hahn-Banach extension theorem in concert with Riesz's representation theorem imply that there exists $w \in L^{p^{\prime}}\left(\Omega, \Lambda^{\ell}\right)$ such that $f(u)=\langle u, w\rangle$ for each $u \in X^{p}\left(\Omega, \Lambda^{\ell}\right)$. Invoking (3.33), we once again decompose $w=w_{1}+w_{2}$ with $w_{1} \in X^{p^{\prime}}\left(\Omega, \Lambda^{\ell}\right)$ and $w_{2} \in Y^{p^{\prime}}\left(\Omega, \Lambda^{\ell}\right)$. Since, as before, $\left\langle u, w_{2}\right\rangle=0$ whenever $u \in X^{p}\left(\Omega, \Lambda^{\ell}\right)$, we may conclude that $\Phi\left(w_{1}\right)=f$. This proves that $\Phi$ is also onto, hence an isomorphism.

Proposition 3.5. For each $p \in\left(p_{\Omega}, q_{\Omega}\right)$, the operator $\mathbb{P}_{p}$ introduced in (3.30) maps

$$
\mathbb{P}_{p}: L^{p}\left(\Omega, \Lambda^{\ell}\right) \longrightarrow X^{p}\left(\Omega, \Lambda^{\ell}\right)
$$

in a bounded, linear fashion, and satisfies

$$
\mathbb{P}_{p}^{2}=\mathbb{P}_{p}, \quad\left(\mathbb{P}_{p}\right)^{*}=\mathbb{P}_{p^{\prime}} \quad \text { if } 1 / p+1 / p^{\prime}=1 .
$$

Proof. The first part in the statement of the proposition follows from the fact that $G_{p}$ maps $L^{p}\left(\Omega, \Lambda^{\ell}\right)$ into $\operatorname{Dom}\left(B_{p}\right)$. As for (3.36), we first note that thanks to (3.21),

$$
I-\mathbb{P}_{p}=d \delta G_{p} .
$$

Based on this, if $u \in L^{p}\left(\Omega, \Lambda^{\ell}\right)$ and $v \in L^{p^{\prime}}\left(\Omega, \Lambda^{\ell}\right)$ with $p_{\Omega}<p, p^{\prime}<q_{\Omega}$, we obtain

$$
\left\langle\mathbb{P}_{p} u,\left(I-\mathbb{P}_{p}\right) v\right\rangle=\left\langle\mathbb{P}_{p}, d \delta G_{p^{\prime}} v\right\rangle=0
$$

where in the last step we have used (2.18) and (3.31). Thus, $\left\langle\mathbb{P}_{p} u, v\right\rangle=\left\langle\mathbb{P}_{p} u, \mathbb{P}_{p^{\prime}} v\right\rangle$ and, since the last expression is symmetric in $u$ and $v$, we may ultimately conclude that $\left\langle\mathbb{P}_{p} u, v\right\rangle=\left\langle u, \mathbb{P}_{p^{\prime}} v\right\rangle$. Hence, $\left(\mathbb{P}_{p}\right)^{*}=\mathbb{P}_{p^{\prime}}$. Armed with this, we may now write $\left\langle\mathbb{P}_{p}^{2} u, v\right\rangle=\left\langle\mathbb{P}_{p} u, \mathbb{P}_{p^{\prime}} v\right\rangle=\left\langle\mathbb{P}_{p} u, v\right\rangle$ so that $\mathbb{P}_{p}^{2}=\mathbb{P}_{p}$, as desired.

Lemma 3.6. For each $p_{\Omega}<p<q_{\Omega}$,

$$
\operatorname{Dom}\left(B_{p}\right) \cap X^{p}\left(\Omega, \Lambda^{\ell}\right)=\left\{u \in \operatorname{Dom}\left(B_{p}\right): B_{p} u \in X^{p}\left(\Omega, \Lambda^{\ell}\right)\right\} .
$$

Proof. Let $u \in \operatorname{Dom}\left(B_{p}\right) \cap X^{p}\left(\Omega, \Lambda^{\ell}\right)$ be arbitrary. Then $B_{p} u=\delta d u$ satisfies $\delta(\delta d u)=0$ and $\nu \vee \delta d u=-\delta_{\partial}(\nu \vee d u)=0$. Thus $B_{p} u \in X^{p}\left(\Omega, \Lambda^{\ell}\right)$, proving the leftto-right inclusion in (3.40). To prove the opposite one, assume that $u \in \operatorname{Dom}\left(B_{p}\right)$ has the property that $B_{p} u \in X^{p}\left(\Omega, \Lambda^{\ell}\right)$. Then $0=\nu \vee(\delta d+d \delta) u=\nu \vee d \delta u$ and $0=\delta(\delta d+d \delta) u=\delta(d \delta u)=\Delta(\delta u)$. Since $\nu \vee \delta u=-\delta_{\partial}(\nu \vee u)=0$, it follows that $\delta u \in \operatorname{Ker}\left(B_{p}\right)=\mathcal{H}_{\vee}^{p}\left(\Omega, \Lambda^{\ell}\right)$, by (3.25). From this we may deduce that $w:=\delta u$ satisfies $d w=0$ and that $w \in L^{q}\left(\Omega, \Lambda^{\ell-1}\right)$ for each $p_{\Omega}<q<q_{\Omega}$. Membership of $u$ to $\operatorname{Dom}\left(B_{p}\right)$ also guarantees that $\nu \vee u=0$. In particular, the integration by parts formula (2.18) applies and gives that $\langle\delta u, \delta u\rangle=\langle\delta u, w\rangle=0$. In turn, this forces $\delta u=0$ which, further, entails $u \in X^{p}\left(\Omega, \Lambda^{\ell}\right)$. 
Lemma 3.7. For each $p_{\Omega}<p<q_{\Omega}$,

$$
\mathbb{P}_{p} B_{p}=B_{p} \mathbb{P}_{p} \text { on } \operatorname{Dom}\left(B_{p}\right) .
$$

Proof. We first claim that if $u \in \operatorname{Dom}\left(B_{p}\right)$, then $d \delta G_{p} u \in \operatorname{Dom}\left(B_{p}\right)$. Indeed,

$$
\begin{aligned}
& d \delta G_{p} u \in L^{p}\left(\Omega, \Lambda^{\ell}\right), \\
& d\left(d \delta G_{p} u\right)=0 \in L^{p}\left(\Omega, \Lambda^{\ell+1}\right), \\
& \delta\left(d \delta G_{p} u\right)=-\delta \Delta G_{p} u=-\delta\left(I-P_{p}\right) u=-\delta u \in L^{p}\left(\Omega, \Lambda^{\ell-1}\right), \\
& (d \delta)\left(d \delta G_{p} u\right)=d\left[\delta\left(d \delta G_{p} u\right)\right]=-d \delta u \in L^{p}\left(\Omega, \Lambda^{\ell}\right), \\
& (\delta d)\left(d \delta G_{p} u\right)=0 \in L^{p}\left(\Omega, \Lambda^{\ell}\right),
\end{aligned}
$$

and $\nu \vee d\left(d \delta G_{p} u\right)=0$,

$$
\nu \vee d \delta G_{p} u=-\nu \delta d G_{p} u-\nu \vee u+\nu \vee P_{p} u=0-\delta_{\partial}\left(\nu \vee d G_{p} u\right)-0=0
$$

justifying the claim. Consequently,

$$
\mathbb{P}_{p} u=u-\delta d G_{p} u \in \operatorname{Dom}\left(B_{p}\right) \text { if } u \in \operatorname{Dom}\left(B_{p}\right),
$$

or, in other words,

$$
\mathbb{P}_{p}: \operatorname{Dom}\left(B_{p}\right) \longrightarrow \operatorname{Dom}\left(B_{p}\right) \cap X^{p}\left(\Omega, \Lambda^{\ell}\right) .
$$

Furthermore, for every $u \in \operatorname{Dom}\left(B_{p}\right)$, we may write

$$
\begin{aligned}
B_{p} \mathbb{P}_{p} u & =-\Delta\left(u-d \delta G_{p} u\right) \\
& =d \delta u+\delta d u+d \delta \Delta G_{p} u=d \delta u+\delta d u+d \delta\left(P_{p}-I\right) u \\
& =\delta d u
\end{aligned}
$$

On the other hand, for every $u \in \operatorname{Dom}\left(B_{p}\right)$,

$$
\mathbb{P}_{p} B_{p} u=\delta d G_{p} B_{p} u+P_{p} B_{p} u=\delta d\left(u-P_{p} u\right)+0=\delta d u,
$$

which, in concert with (3.46), proves (3.41).

Lemma 3.8. If $p_{\Omega}<p<q_{\Omega}$, then for each $0 \leq \ell \leq n$,

$$
d=d \mathbb{P}_{p, \ell}+\mathbb{P}_{p, \ell+1} d \text { on } \mathcal{D}_{\ell}^{p}(\Omega ; d),
$$

where $\mathbb{P}_{p, \ell}$ stands for $\mathbb{P}_{p}$ acting on $L^{p}\left(\Omega, \Lambda^{\ell}\right)$, etc.

Proof. Since $P_{p, \ell+1}(d u)=0$ and $d P_{p, \ell} u=0$ for every $u \in \mathcal{D}_{\ell}^{p}(\Omega ; d)$, based on (3.30), (3.24) and (3.21) we may write

$$
\begin{aligned}
d \mathbb{P}_{p, \ell} u & =d \delta G_{p, \ell+1}(d u)=-\delta d G_{p, \ell+1}(d u)-\Delta G_{p, \ell+1}(d u) \\
& =-\mathbb{P}_{p, \ell+1}(d u)+d u, \quad \forall u \in \mathcal{D}_{\ell}^{p}(\Omega ; d),
\end{aligned}
$$

from which (3.48) follows. 
3.2. The Stokes operator. Given a Lipschitz subdomain $\Omega$ of $\mathcal{M}$ and $1<p<\infty$, let us now define the Stokes operator

$$
A_{p}: X^{p}\left(\Omega, \Lambda^{\ell}\right) \longrightarrow X^{p}\left(\Omega, \Lambda^{\ell}\right)
$$

by setting

$$
\begin{aligned}
& \operatorname{Dom}\left(A_{p}\right):=\operatorname{Dom}\left(B_{p}\right) \cap X^{p}\left(\Omega, \Lambda^{\ell}\right), \\
& A_{p} u:=B_{p} u=-\Delta u, \quad \forall u \in \operatorname{Dom}\left(A_{p}\right) .
\end{aligned}
$$

Lemma 3.9. For each $p_{\Omega}<p<q_{\Omega}$,

$$
A_{p} \mathbb{P}_{p}=\mathbb{P}_{p} B_{p} \text { on } \operatorname{Dom}\left(B_{p}\right) .
$$

Proof. By relying on Lemma 3.7 and the fact that $A_{p}=B_{p}$ on $\operatorname{Dom}\left(B_{p}\right) \cap$ $X^{p}\left(\Omega, \Lambda^{\ell}\right)$, on $\operatorname{Dom}\left(B_{p}\right)$ we may write

$$
A_{p} \mathbb{P}_{p}=B_{p} \mathbb{P}_{p}=\mathbb{P}_{p} B_{p},
$$

where (3.45) is also used.

For a (possibly unbounded) operator $T$ we let $\operatorname{Spec}(T)$ denote its spectrum.

Lemma 3.10. For each $p_{\Omega}<p<q_{\Omega}$,

$$
\operatorname{Spec}\left(A_{p}\right) \subseteq \operatorname{Spec}\left(B_{p}\right)
$$

and, for each $\lambda \notin \operatorname{Spec}\left(B_{p}\right)$,

$$
\left(\lambda I-B_{p}\right)^{-1} \mathbb{P}_{p}=\mathbb{P}_{p}\left(\lambda I-B_{p}\right)^{-1}=\left(\lambda I-A_{p}\right)^{-1} \mathbb{P}_{p}
$$

on $L^{p}\left(\Omega, \Lambda^{\ell}\right)$.

Proof. If $\lambda \notin \operatorname{Spec}\left(B_{p}\right)$, then $\left(\lambda I-B_{p}\right)^{-1}$ is invertible on $L^{p}\left(\Omega, \Lambda^{\ell}\right)$ and, hence, $\lambda I-A_{p}$ is one-to-one. Next, if $f \in X^{p}\left(\Omega, \Lambda^{\ell}\right) \hookrightarrow L^{p}\left(\Omega, \Lambda^{\ell}\right)$ is arbitrary, consider $w:=\left(\lambda I-B_{p}\right)^{-1} f \in \operatorname{Dom}\left(B_{p}\right) \hookrightarrow L^{p}\left(\Omega, \Lambda^{\ell}\right)$. If we now set $u:=\mathbb{P}_{p} w$ it follows that $u \in \operatorname{Dom}\left(B_{p}\right) \cap X^{p}\left(\Omega, \Lambda^{\ell}\right)=\operatorname{Dom}\left(A_{p}\right)$ and

$$
\left(\lambda I-A_{p}\right)^{-1} u=\lambda \mathbb{P}_{p} w-A_{p} \mathbb{P}_{p} w=\mathbb{P}_{p}\left(\lambda I-B_{p}\right) w=\mathbb{P}_{p} f=f,
$$

which proves that $\lambda I-A_{p}$ is onto as well. Hence, (3.54) holds. Then the commutation identities in (3.55) are straightforward consequences of this and Lemma 3.8 .

Lemma 3.11. Whenever $p_{\Omega}<p, p^{\prime}<q_{\Omega}$ are such that $1 / p+1 / p^{\prime}=1$, it follows that

$$
\left(A_{p}\right)^{*}=A_{p^{\prime}} .
$$

Proof. With $p, p^{\prime}$ as in the statement of the lemma, let $u \in \operatorname{Dom}\left(A_{p}^{*}\right) \subset X^{p^{\prime}}\left(\Omega, \Lambda^{\ell}\right)$ and set $w:=A_{p}^{*} u \in X^{p^{\prime}}\left(\Omega, \Lambda^{\ell}\right)$. In particular,

$$
\langle w, \eta\rangle=\left\langle u, A_{p} \eta\right\rangle, \quad \forall \eta \in \operatorname{Dom}\left(A_{p}\right) \subset X^{p}\left(\Omega, \Lambda^{\ell}\right) .
$$

Then for every $\xi \in \operatorname{Dom}\left(B_{p}\right)$ we may write

$$
\begin{aligned}
\left\langle B_{p} \xi, u\right\rangle & =\left\langle B_{p} \xi, \mathbb{P}_{p^{\prime}} u\right\rangle=\left\langle\mathbb{P}_{p} B_{p} \xi, u\right\rangle \\
& =\left\langle A_{p}\left(\mathbb{P}_{p} \xi\right), u\right\rangle=\left\langle\mathbb{P}_{p} \xi, w\right\rangle=\left\langle\xi, \mathbb{P}_{p^{\prime}} w\right\rangle \\
& =\langle\xi, w\rangle,
\end{aligned}
$$

where we have used (3.58) and the fact that $\mathbb{P}_{p} \xi \in \operatorname{Dom}\left(A_{p}\right)$ whenever $\xi \in$ $\operatorname{Dom}\left(B_{p}\right)$. As a consequence of (3.59) we have $u \in \operatorname{Dom}\left(B_{p}^{*}\right)=\operatorname{Dom}\left(B_{p^{\prime}}\right)$ and, 
moreover, $B_{p^{\prime}} u=w \in X^{p^{\prime}}\left(\Omega, \Lambda^{\ell}\right)$. All in all, $u \in \operatorname{Dom}\left(B_{p^{\prime}}\right) \cap X^{p^{\prime}}\left(\Omega, \Lambda^{\ell}\right)=$ $\operatorname{Dom}\left(A_{p^{\prime}}\right)$ and $A_{p^{\prime}} u=B_{p^{\prime}} u=w=A_{p}^{*} u$. In particular, this shows that $\left(A_{p}\right)^{*} \subseteq$ $A_{p^{\prime}}$.

Conversely, fix an arbitrary $u \in \operatorname{Dom}\left(A_{p^{\prime}}\right)$. Hence, $u \in X^{p^{\prime}}\left(\Omega, \Lambda^{\ell}\right) \cap \operatorname{Dom}\left(B_{p^{\prime}}\right)$, which then gives

$$
\left\langle A_{p^{\prime}} u, w\right\rangle=\left\langle B_{p^{\prime}} u, w\right\rangle=\left\langle u, B_{p} w\right\rangle=\left\langle u, A_{p} w\right\rangle
$$

for each $w \in X^{p}\left(\Omega, \Lambda^{\ell}\right) \cap \operatorname{Dom}\left(B_{p}\right)=\operatorname{Dom}\left(A_{p}\right)$. In turn, this allows us to conclude that $u \in \operatorname{Dom}\left(A_{p}^{*}\right)$ and $A_{p}^{*}=A_{p^{\prime}} u$ and, further, that $A_{p^{\prime}} \subseteq A_{p}^{*}$. The proof of the lemma is therefore finished.

Remark. For each $p_{\Omega}<p<q_{\Omega}$,

$$
\operatorname{Ker} A_{p}=\operatorname{Ker} B_{p}=\mathcal{H}^{p}\left(\Omega, \Lambda^{\ell}\right),
$$

so both operators are invertible if $b_{\ell}(\Omega)=0$.

Proposition 3.12. Let $\ell \in\{0,1, \ldots, n\}$ be fixed and consider the spaces

$$
\mathcal{H}:=X^{2}\left(\Omega, \Lambda^{\ell}\right), \quad \mathcal{V}:=\left\{u \in \mathcal{H}: d u \in L^{2}\left(\Omega, \Lambda^{\ell+1}\right)\right\},
$$

where the latter space is equipped with the natural graph norm. Also, consider the sesquilinear form

$$
a(u, v):=\langle d u, d v\rangle, \quad u, v \in \mathcal{V} .
$$

Then the operator associated with the triplet $\{\mathcal{H}, \mathcal{V}$, a $\}$ as in $\S 2.3$ is precisely $A_{2}$ (i.e., the Stokes operator introduced in (3.50)-(3.51) for $p=2$ ). In particular, $A_{2}$ generates an analytic semigroup on $X^{2}\left(\Omega, \Lambda^{\ell}\right)$.

Proof. According to the discussion in $\S 2.3$, the domain of the operator $A$ associated with the triplet $\{\mathcal{H}, \mathcal{V}, a\}$ consists of forms $u \in \mathcal{V}$ for which there exists $w \in \mathcal{H}$ such that

$$
\langle d u, d v\rangle=\langle w, v\rangle, \quad \forall v \in \mathcal{V} .
$$

In order to continue, we shall need the fact that the operator

$$
\mathbb{P}_{2}: \mathcal{D}_{\ell}^{2}(\Omega ; d) \longrightarrow \mathcal{V} \quad \text { is onto. }
$$

Indeed, the fact that $\mathbb{P}_{2}$ maps the space $\mathcal{D}_{\ell}^{2}(\Omega ; d)$ into $\mathcal{V}$ is clear from Lemma 3.8 . Since

$$
\mathbb{P}_{2, \ell} \text { acts as the identity on } \mathcal{H},
$$

any $v \in \mathcal{V}=\mathcal{H} \cap \mathcal{D}_{\ell}^{2}(\Omega ; d)$ can be written as $v=\mathbb{P}_{2} v$, proving (3.65).

Returning now to the mainstream discussion, the above analysis shows that if $u, w$ are as in the opening paragraph, then condition (3.64) is equivalent to

$$
\left\langle d u, d \mathbb{P}_{2} f\right\rangle=\left\langle w, \mathbb{P}_{2} f\right\rangle, \quad \forall f \in \mathcal{D}_{\ell}^{2}(\Omega ; d) .
$$

Now, if $f \in \mathcal{D}_{\ell}^{2}(\Omega ; d)$ is arbitrary, based on this, (3.66) and (3.49) we may write

$$
\begin{aligned}
\langle w, f\rangle & =\left\langle\mathbb{P}_{2} w, f\right\rangle=\left\langle w, \mathbb{P}_{2} f\right\rangle=\left\langle d u, d \mathbb{P}_{2} f\right\rangle \\
& =\left\langle d u,-\mathbb{P}_{2, \ell+1}(d f)+d f\right\rangle=\left\langle d u,-\mathbb{P}_{2, \ell+1}(d f)\right\rangle+\langle d u, d f\rangle \\
& =\left\langle-\mathbb{P}_{2, \ell+1}(d u), d f\right\rangle+\langle d u, d f\rangle=\left\langle-\mathbb{P}_{2, \ell+1}(d u)+d u, d f\right\rangle \\
& =\left\langle d \mathbb{P}_{2, \ell} u, d f\right\rangle=\langle d u, d f\rangle .
\end{aligned}
$$

In turn, this is equivalent to demanding that $d u \in \mathcal{D}_{\ell+1}^{2}\left(\Omega ; \delta_{\vee}\right)$ and $\delta d u=w$. 
Consequently, the domain of $A$ is precisely the collection of forms (3.69)

$$
u \in L^{2}\left(\Omega, \Lambda^{\ell}\right), \quad \delta u=0, \quad \nu \vee u=0, \quad d u \in L^{2}\left(\Omega, \Lambda^{\ell+1}\right), \quad \delta d u \in L^{2}\left(\Omega, \Lambda^{\ell}\right),
$$

and $A u=\delta d u=-\Delta u$ for each $u$ as in (3.69). That is, $A$ coincides with $A_{2}$ (introduced in (3.50)-(3.51)), as desired.

3.3. The Maxwell operator. For an arbitrary Lipschitz subdomain $\Omega$ of $\mathcal{M}$ and $p_{\Omega}<p<q_{\Omega}$, we introduce the spaces

$$
\begin{aligned}
& Z^{p}\left(\Omega, \Lambda^{\ell}\right):=\left\{u \in L^{p}\left(\Omega, \Lambda^{\ell}\right): d u=0\right\}, \\
& W^{p}\left(\Omega, \Lambda^{\ell}\right):=\left\{\delta u: u \in \mathcal{D}_{\ell}^{p}\left(\Omega ; \delta_{\vee}\right)\right\},
\end{aligned}
$$

and consider the operator

$$
\mathbb{Q}_{p}:=d \delta G_{p}+P_{p}: L^{p}\left(\Omega, \Lambda^{\ell}\right) \longrightarrow L^{p}\left(\Omega, \Lambda^{\ell}\right) .
$$

Its main properties are summarized in the lemma below.

Lemma 3.13. For each $p_{\Omega}<p<q_{\Omega}$, the following hold:

(i) $\left(\mathbb{Q}_{p}\right)^{*}=\mathbb{Q}_{p^{\prime}}$ if $1 / p+1 / p^{\prime}=1$, and $\left(\mathbb{Q}_{p}\right)^{2}=\mathbb{Q}_{p}$;

(ii) $\mathbb{Q}_{p} B_{p}=B_{p} \mathbb{Q}_{p}$ on $\operatorname{Dom}\left(B_{p}\right)$;

(iii) $\mathbb{Q}_{p}: L^{p}\left(\Omega, \Lambda^{\ell}\right) \rightarrow Z^{p}\left(\Omega, \Lambda^{\ell}\right)$ is onto;

(iv) $\mathbb{Q}_{p}: \mathcal{D}_{\ell}^{p}\left(\Omega ; \delta_{\vee}\right) \rightarrow Z^{p}\left(\Omega, \Lambda^{\ell}\right) \cap \mathcal{D}_{\ell}^{p}\left(\Omega ; \delta_{\vee}\right)$ is onto;

(v) $\delta=\delta \mathbb{Q}_{p, \ell}+\mathbb{Q}_{p, \ell-1} \delta$ on $\mathcal{D}_{\ell}^{p}\left(\Omega ; \delta_{\vee}\right)$.

We omit the proof, which can be carried out much as for the case of the Leray projection $\mathbb{P}_{p}$.

Next, for each $p_{\Omega}<p<q_{\Omega}$, the Maxwell operator is introduced as the part of $B_{p}$ in $Z^{p}\left(\Omega, \Lambda^{\ell}\right)$, i.e., as the unbounded operator

$$
C_{p}: Z^{p}\left(\Omega, \Lambda^{\ell}\right) \longrightarrow Z^{p}\left(\Omega, \Lambda^{\ell}\right)
$$

for which

$$
\begin{aligned}
& \operatorname{Dom}\left(C_{p}\right):=\operatorname{Dom}\left(B_{p}\right) \cap Z^{p}\left(\Omega, \Lambda^{\ell}\right), \\
& C_{p} u:=B_{p} u=-\Delta u, \quad \forall u \in \operatorname{Dom}\left(C_{p}\right) .
\end{aligned}
$$

Some of the most basic properties of this operator are summarized below. They parallel those of the Stokes operator discussed in $\S 3.2$ and can be proved in much the same fashion.

Lemma 3.14. For each $p_{\Omega}<p<q_{\Omega}$, the following hold.

(i) $C_{p} \mathbb{Q}_{p}=\mathbb{Q}_{p} B_{p}$ on $\operatorname{Dom}\left(B_{p}\right)$;

(ii) $\operatorname{Spec}\left(C_{p}\right) \subseteq \operatorname{Spec}\left(B_{p}\right)$ and, for each $\lambda \notin \operatorname{Spec}\left(B_{p}\right)$,

$$
\left(\lambda I-B_{p}\right)^{-1} \mathbb{Q}_{p}=\mathbb{Q}_{p}\left(\lambda I-B_{p}\right)^{-1}=\left(\lambda I-C_{p}\right)^{-1} \mathbb{Q}_{p} \text { on } L^{p}\left(\Omega, \Lambda^{\ell}\right) ;
$$

(iii) $\left(Z^{p}\left(\Omega, \Lambda^{\ell}\right)\right)^{*}=Z^{p^{\prime}}\left(\Omega, \Lambda^{\ell}\right)$ and $\left(C_{p}\right)^{*}=C_{p^{\prime}}$ whenever $1 / p+1 / p^{\prime}=1$;

(iv) $\operatorname{Ker} C_{p}=\operatorname{Ker} B_{p}=\mathcal{H}^{p}\left(\Omega, \Lambda^{\ell}\right)$ so that, in particular, the operator (3.72)(3.73) is invertible if $b_{\ell}(\Omega)=0$.

Finally, we remark that, in the case when $p=2$, the Maxwell operator (3.72)(3.73) generates an analytic semigroup on $Z^{2}\left(\Omega, \Lambda^{\ell}\right)$. More specifically, we have the following. 
Proposition 3.15. Fix $\ell \in\{0,1, \ldots, n\}$ and consider the spaces

$$
\mathcal{H}:=Z^{2}\left(\Omega, \Lambda^{\ell}\right), \quad \mathcal{V}:=\left\{u \in \mathcal{H}: \delta u \in L^{2}\left(\Omega, \Lambda^{\ell+1}\right), \nu \vee u=0\right\},
$$

where the latter space is equipped with the natural graph norm. Also, consider the sesquilinear form

$$
a(u, v):=\langle\delta u, \delta v\rangle, \quad u, v \in \mathcal{V} .
$$

Then the operator associated with the triplet $\{\mathcal{H}, \mathcal{V}, a\}$ as in $\S 2.3$ is precisely the Maxwell operator $C_{2}$. In particular, $C_{2}$ generates an analytic semigroup on $Z^{2}\left(\Omega, \Lambda^{\ell}\right)$.

The proof of these results can be carried out much as the proof of Proposition 3.12 with the help of Lemma 3.14.

\section{The Regularity of Differential forms in $\operatorname{Dom}\left(B_{p}\right)$}

We first record a number of useful results from [19, 20. For each $0 \leq \ell \leq n$, the operator $-\Delta_{\ell}=-\Delta: L_{1}^{2}\left(\mathcal{M}, \Lambda^{\ell}\right) \rightarrow L_{-1}^{2}\left(\mathcal{M}, \Lambda^{\ell}\right)$ is bounded, nonnegative, and selfadjoint. Since for $\lambda \in \mathbb{R}$ with $\lambda>0$, the operator $\left(\lambda I-\Delta_{\ell}\right)^{-1}$ is positive, selfadjoint and compact on $L^{2}\left(\mathcal{M}, \Lambda^{\ell}\right)$ it follows that there exists $\operatorname{Spec}\left(\Delta_{\ell}\right) \subseteq(-\infty, 0]$, a discrete set such that

$$
z \notin \operatorname{Spec}\left(\Delta_{\ell}\right) \Rightarrow\left(\Delta_{\ell}-z I\right): L_{1}^{2}\left(\mathcal{M}, \Lambda^{\ell}\right) \longrightarrow L_{-1}^{2}\left(\mathcal{M}, \Lambda^{\ell}\right) \text { is invertible. }
$$

Set

$$
\mathcal{U}:=\bigcup_{0 \leq \ell \leq n} \operatorname{Spec}\left(\Delta_{\ell}\right) \subset(-\infty, 0]
$$

and for $\lambda \notin \mathcal{U}$, let $\Gamma_{\lambda, \ell}$ be the Schwartz kernel of $\Delta-\lambda I$ on $\ell$-forms. In particular, we denote by $\Pi_{\lambda, \ell}$ the associated (volume) Newtonian potential. Also, once a Lipschitz domain $\Omega \subset \mathcal{M}$ has been fixed, we define the single layer potential operator on $\partial \Omega$ by

$$
\mathcal{S}_{\lambda, \ell} f(x):=\int_{\partial \Omega}\left\langle\Gamma_{\lambda, \ell}(x, y), f(y)\right\rangle d \sigma_{y}, \quad x \in \Omega,
$$

for any $f: \partial \Omega \rightarrow \Lambda^{\ell}$. Note that $(\Delta-\lambda I) \mathcal{S}_{\lambda, \ell}=0$ in $\Omega$ and, as proved in [19], the operators

$$
\begin{aligned}
& \mathcal{S}_{\lambda, \ell}: \mathcal{X}_{\ell}^{p}(\partial \Omega) \longrightarrow L_{1}^{p}\left(\Omega, \Lambda^{\ell}\right), \\
& d \mathcal{S}_{\lambda, \ell}: \mathcal{X}_{\ell}^{p}(\partial \Omega) \longrightarrow \mathcal{D}_{\ell+1}^{p}(\Omega ; \delta),
\end{aligned}
$$

are well-defined and bounded. As a consequence, the operator $M_{\lambda, \ell}$ defined by the equality

$$
\left(-\frac{1}{2} I+M_{\lambda, \ell}\right) f=\nu \vee\left(d \mathcal{S}_{\lambda, \ell} f\right), \quad \forall f \in \mathcal{X}_{\ell}^{p}(\partial \Omega),
$$

is well-defined and bounded on $\mathcal{X}_{\ell}^{p}(\partial \Omega)$ for each $p \in(1, \infty)$. It has also been shown in [19] that if $0 \leq \ell \leq n, \lambda \notin \mathcal{U}$ and $p_{\Omega}<p<q_{\Omega}$,

$$
-\frac{1}{2} I+M_{\lambda, \ell}: \mathcal{X}_{\ell}^{p}(\partial \Omega) \longrightarrow \mathcal{X}_{\ell}^{p}(\partial \Omega) \text { is an isomorphism. }
$$


Our final remark is that the spectrum of $B_{p}$ acting on $L^{p}\left(\Omega, \Lambda^{\ell}\right)$ is a discrete subset $\operatorname{Spec}\left(B_{\ell}\right)$ of $(-\infty, 0]$ which is independent of $p \in\left(p_{\Omega}, q_{\Omega}\right)$; cf. [19]. We then set

$$
\mathcal{U}_{o}:=\mathcal{U} \cup\left(\bigcup_{0 \leq \ell \leq n} \operatorname{Spec}\left(B_{\ell}\right)\right)
$$

Proposition 4.1. Assume that $\lambda \in \mathbb{C} \backslash \mathcal{U}_{o}$ and that $p_{\Omega}<p<q<q_{\Omega}$. Then, if $u \in \operatorname{Dom}\left(B_{p}\right)$ is such that $(\lambda I-\Delta) u \in L^{q}\left(\Omega, \Lambda^{\ell}\right)$, it follows that $u \in \operatorname{Dom}\left(B_{q}\right)$.

Proof. Denote by $\tilde{f}$ the extension of $f:=(\lambda I-\Delta) u \in L^{q}\left(\Omega, \Lambda^{\ell}\right)$ by zero in $\mathcal{M} \backslash \Omega$ and set

$$
\eta:=\left.\left[(\Delta-\lambda I)^{-1} \tilde{f}\right]\right|_{\Omega} \in L_{2}^{q}\left(\Omega, \Lambda^{\ell}\right) .
$$

Then the differential form

$$
\begin{aligned}
& w:=\mathcal{S}_{\lambda, \ell}\left[\left(-\frac{1}{2} I+M_{\lambda, \ell}\right)^{-1}(\nu \vee d \eta)\right] \\
& +d \mathcal{S}_{\lambda, \ell-1}\left\{\left(-\frac{1}{2} I+M_{\lambda, \ell-1}\right)^{-1}\left(\nu \vee \eta-\nu \vee \mathcal{S}_{\lambda, \ell}\left[\left(-\frac{1}{2} I+M_{\lambda, \ell}\right)^{-1}(\nu \vee d \eta)\right]\right)\right\}
\end{aligned}
$$

satisfies, thanks to (4.4), (4.5), (4.7), and the fact that $d \delta=-\Delta-\delta d$,

$$
\left\{\begin{array}{l}
(\Delta-\lambda I) w=0 \text { in } \Omega \\
w, d \delta w, \delta d w \in L^{q}\left(\Omega, \Lambda^{\ell}\right) \\
d w \in L^{q}\left(\Omega, \Lambda^{\ell+1}\right), \delta w \in L^{q}\left(\Omega, \Lambda^{\ell-1}\right) \\
\nu \vee w=\nu \vee \eta \\
\nu \vee d w=\nu \vee d \eta
\end{array}\right.
$$

It follows that $w-\eta \in \operatorname{Dom}\left(B_{q}\right) \hookrightarrow \operatorname{Dom}\left(B_{p}\right)$ and $\left(\lambda I+B_{p}\right)(w-\eta)=f$. Consequently, $u=\left(\lambda I+B_{p}\right)^{-1} f=w-\eta \in \operatorname{Dom}\left(B_{q}\right)$, as claimed.

Our last result in this section can, informally speaking, be regarded as a statement about the $L^{p}$-boundedness of the Riesz transforms $d \delta \Delta^{-1}, \delta d \Delta^{-1}$. Alternatively, it is a statement about the maximal regularity of $-\Delta$ relative to the decomposition $-\Delta=\delta d+d \delta$.

Proposition 4.2. Assume that $\Omega$ is a Lipschitz subdomain of the manifold $\mathcal{M}$. If $p_{\Omega}<p<q_{\Omega}$ and $u \in \operatorname{Dom}\left(B_{p}\right)$, then

$$
\|d \delta u\|_{L^{p}\left(\Omega, \Lambda^{\ell}\right)}+\|\delta d u\|_{L^{p}\left(\Omega, \Lambda^{\ell}\right)} \leq C\|\Delta u\|_{L^{p}\left(\Omega, \Lambda^{\ell}\right)}
$$

for some finite $C=C(\partial \Omega, p)>0$.

Proof. Let the index $p$ and the differential form $u$ be as in the statement of the proposition and set $f:=\Delta u \in L^{p}\left(\Omega, \Lambda^{\ell}\right)$. Applying $G_{p}$ to this equality and relying on (3.21) leads to the conclusion that $u=G_{p} f+P_{p} u$. Hence, $d \delta u=d \delta G_{p} f$ and $\delta d u=\delta d G_{p} f$. Having justified this representation, the estimate (4.11) is a direct consequence of (3.20). 


\section{Main Lemma}

For an arbitrary, fixed $\theta \in(0, \pi)$, consider the sector

$$
\Sigma_{\theta}:=\{z \in \mathbb{C}:|\arg z|<\pi-\theta\} \subset \mathbb{C}
$$

and note that, generally speaking,

$$
|\lambda a+b| \approx|\lambda| a+b, \text { uniformly for } \lambda \in \Sigma_{\theta}, a, b \geq 0 .
$$

In what follows, we shall work with the convention that

$$
1<p<\infty \Longrightarrow p^{*}:=\frac{n p}{n-1} \text {. }
$$

Besides these conventions, below we collect a number of hypotheses which we will assume to be valid throughout this section.

Hypotheses. Consider an arbitrary $\theta \in(0, \pi)$, and arbitrary $\lambda \in \Sigma_{\theta}$ and set

$$
t:=\frac{1}{\sqrt{|\lambda|}}=|\lambda|^{-1 / 2}
$$

For a fixed $\ell \in\{0,1, \ldots, n\}$, consider an arbitrary form

$$
f \in C_{0}^{\infty}\left(\Omega, \Lambda^{\ell}\right) \hookrightarrow L^{2}\left(\Omega, \Lambda^{\ell}\right)
$$

and define

$$
u:=\left(\lambda I+B_{2}\right)^{-1} f \in \operatorname{Dom}\left(B_{2}\right) \hookrightarrow L^{2}\left(\Omega, \Lambda^{\ell}\right) .
$$

Next, fix an arbitrary point $x \in \Omega$, an arbitrary sequence of functions $\left\{\eta_{j}\right\}_{j \geq 0}$ such that

$$
\begin{aligned}
& \eta_{0} \in C_{o}^{\infty}(B(x, 2 t)), \quad \eta_{j} \in C_{o}^{\infty}\left(B\left(x, 2^{j+1} t\right) \backslash B\left(x, 2^{j-1} t\right)\right) \\
& 0 \leq \eta_{j} \leq 1, \quad\left|\nabla \eta_{j}\right| \leq \frac{1}{2^{j-1} t}, \quad \sum_{j=0}^{\infty} \eta_{j}=1
\end{aligned}
$$

and decompose

$$
\begin{aligned}
& f=\sum_{j=0}^{\infty} f_{j}, \quad f_{j}:=\eta_{j} f \in L^{p}\left(\Omega, \Lambda^{\ell}\right) \hookrightarrow L^{2}\left(\Omega, \Lambda^{\ell}\right), \\
& u=\sum_{j=0}^{\infty} u_{j}, \quad u_{j}:=\left(\lambda I+B_{2}\right)^{-1} f_{j} \in \operatorname{Dom}\left(B_{2}\right),
\end{aligned}
$$

where the embedding in (5.8) presupposes that $p \geq 2$ (see below).

Going further, assume that there exist

$$
2 \leq p<q_{\Omega}
$$

and two finite constants $C, c>0$ with the property that

$$
\begin{aligned}
|\lambda|\left[\int_{\Omega \cap B(x, t)}\left|u_{j}\right|^{p} d V\right]^{1 / p}+|\lambda|^{1 / 2}\left[\int_{\Omega \cap B(x, t)}\left|d u_{j}\right|^{p} d V\right]^{1 / p} \\
\quad+|\lambda|^{1 / 2}\left[\int_{\Omega \cap B(x, t)}\left|\delta u_{j}\right|^{p} d V\right]^{1 / p} \leq C e^{-c 2^{j}}\left[\int_{\Omega}\left|f_{j}\right|^{p} d V\right]^{1 / p}
\end{aligned}
$$


for each $j \geq 0$, and such that

$$
\begin{gathered}
{\left[\int_{\Omega \cap B(x, t)}\left|\delta d u_{j}\right|^{p} d V\right]^{1 / p}+\left[\int_{\Omega \cap B(x, t)}\left|d \delta u_{j}\right|^{p} d V\right]^{1 / p}} \\
\leq C e^{-c 2^{j}}\left[\int_{\Omega}\left|f_{j}\right|^{p} d V\right]^{1 / p}, \quad \forall j \geq 0 .
\end{gathered}
$$

It is illuminating to note that the above are in effect high-energy exponential decay estimates of the support of the inhomogeneous term, $f_{j}$. The above decomposition of $f$ into the pieces $f_{j}$ takes into account the natural high-energy scaling (roughly, $\sqrt{|\lambda|}|y-x|$ if one localizes the problem in $y$ near $x$ ), and is inspired by the work in [2, in connection with Kato's square-root problem.

Lemma 5.1. Granted the above conventions and assumptions, for each $k \in \mathbb{N}$ there exist two finite constants $C, c>0$ depending only on $\theta, p$ and the Lipschitz character of $\Omega$ such that, for each $j \geq 0$,

$$
\begin{aligned}
|\lambda|\left[\int_{\Omega \cap B(x, t)}\left|u_{j}\right|^{p^{*}} d V\right]^{1 / p^{*}}+|\lambda|^{1 / 2}\left[\int_{\Omega \cap B(x, t)}\left|d u_{j}\right|^{p^{*}} d V\right]^{1 / p^{*}} \\
\quad+|\lambda|^{1 / 2}\left[\int_{\Omega \cap B(x, t)}\left|\delta u_{j}\right|^{p^{*}} d V\right]^{1 / p^{*}} \leq C e^{-c 2^{j}}\left[\int_{\Omega}\left|f_{j}\right|^{p^{*}} d V\right]^{1 / p^{*}},
\end{aligned}
$$

and, if in addition to the hypotheses made so far we also have

$$
p^{*}<q_{\Omega},
$$

then also

$$
\begin{gathered}
{\left[\int_{\Omega \cap B(x, t)}\left|\delta d u_{j}\right|^{p^{*}} d V\right]^{1 / p^{*}}+\left[\int_{\Omega \cap B(x, t)}\left|d \delta u_{j}\right|^{p^{*}} d V\right]^{1 / p^{*}}} \\
\leq C e^{-c 2^{j}}\left[\int_{\Omega}\left|f_{j}\right|^{p^{*}} d V\right]^{1 / p^{*}}, \quad \forall j \geq 0 .
\end{gathered}
$$

Proof. To prove this, we shall assume (5.7)-(5.12) and proceed in a series of steps starting with

Step 1. For each $j \geq 0$,

$$
\begin{array}{ll}
u_{j} \in L^{2}\left(\Omega, \Lambda^{\ell}\right), \quad d u_{j} \in L^{2}\left(\Omega, \Lambda^{\ell+1}\right), & \delta u_{j} \in L^{2}\left(\Omega, \Lambda^{\ell-1}\right), \\
\delta d u_{j}, d \delta u_{j} \in L^{2}\left(\Omega, \Lambda^{\ell}\right), \quad \nu \vee u_{j}=0, \quad \nu \vee d u_{j}=0,
\end{array}
$$

and

$$
\lambda u_{j}-\Delta u_{j}=f_{j} \quad \text { in } \Omega .
$$

These follow from the definition of $u_{j} \in \operatorname{Dom}\left(B_{2}\right)$.

Step 2. For any Lipschitz subdomain $D$ of $\mathcal{M}$ there exists $2<q_{D} \leq \infty$ with the following significance. For each $p \in\left[2, q_{D}\right)$ there exists $C>0$ which depends exclusively on $p$ and the Lipschitz character of $D$ such that for any $w$ satisfying

$$
\begin{aligned}
& w \in L^{p}\left(D, \Lambda^{\ell}\right), \quad d w \in L^{p}\left(D, \Lambda^{\ell+1}\right), \quad \delta w \in L^{p}\left(D, \Lambda^{\ell-1}\right) \\
& \text { and such that } \nu \vee w=0 \quad \text { on } \partial D,
\end{aligned}
$$


it follows that

$$
\begin{aligned}
& R^{n\left(\frac{1}{p}-\frac{1}{p^{*}}\right)}\left[\int_{D}|w|^{p^{*}} d V\right]^{1 / p^{*}} \\
& \quad \leq C\left\{\left[\int_{D}|w|^{p} d V\right]^{1 / p}+R\left[\int_{D}|d w|^{p} d V\right]^{1 / p}+R\left[\int_{D}|\delta w|^{p} d V\right]^{1 / p}\right\}
\end{aligned}
$$

where $R:=\operatorname{diam}(D)$ and, as before, $p^{*}:=n p /(n-1)$.

To justify this, we first recall that, under the assumptions (5.18), the estimate

$$
\|w\|_{B_{1, p}^{p, p}\left(D, \Lambda^{\ell}\right)} \leq C\left[\|w\|_{L^{p}\left(D, \Lambda^{\ell}\right)}+\|d w\|_{L^{p}\left(D, \Lambda^{\ell+1}\right)}+\|\delta w\|_{L^{p}\left(D, \Lambda^{\ell-1}\right)}\right]
$$

has been established in [18, for a constant $C=C(\partial D$, diam $D)>0$ independent of $w$. Now (5.19) follows from this, Sobolev's embedding theorem to the effect that

$$
B_{1 / p}^{p, p}(\Omega) \hookrightarrow L^{p^{*}}(\Omega), \quad p^{*}=\frac{n p}{n-1},
$$

and rescaling.

Step 3. For each $k \in \mathbb{N}$ there exist $C, c>0$ such that

$$
|\lambda|\left[\int_{\Omega \cap B(x, t)}\left|u_{j}\right|^{p^{*}} d V\right]^{1 / p^{*}} \leq C e^{-c 2^{j}} t^{n\left(\frac{1}{p^{*}}-\frac{1}{p}\right)}\left[\int_{\Omega}\left|f_{j}\right|^{p} d V\right]^{1 / p}
$$

and

$$
|\lambda|\left[\int_{\Omega \cap B(x, t)}\left|u_{j}\right|^{p^{*}} d V\right]^{1 / p^{*}} \leq C e^{-c 2^{j}}\left[\int_{\Omega}\left|f_{j}\right|^{p^{*}} d V\right]^{1 / p^{*}}
$$

for each $j \geq 0$.

To prove this, fix a function

$$
\zeta \in C_{o}^{\infty}(B(x, t)) \text { with } \zeta \equiv 1 \text { on } B(x, t / 2) \text { and }|\nabla \zeta| \leq C t^{-1}
$$

and use (5.19) in the context when $D:=\Omega \cap B(x, t)$ and $w:=\zeta u_{j}$. That this applies is guaranteed by the fact that $\nu \vee\left(\zeta u_{j}\right)=0$ on $\partial[\Omega \cap B(x, t)]$ and

$$
\begin{aligned}
\left\|\zeta u_{j}\right\|_{L^{p}\left(\Omega \cap B(x, t), \Lambda^{\ell}\right)} \leq\left\|u_{j}\right\|_{L^{p}\left(\Omega \cap B(x, t), \Lambda^{\ell}\right)}, & \\
\left\|d\left(\zeta u_{j}\right)\right\|_{L^{p}\left(\Omega \cap B(x, t), \Lambda^{\ell+1}\right)} \leq & C t^{-1}\left\|u_{j}\right\|_{L^{p}\left(\Omega \cap B(x, t), \Lambda^{\ell}\right)} \\
& +\left\|d u_{j}\right\|_{L^{p}\left(\Omega \cap B(x, t), \Lambda^{\ell+1}\right)}, \\
\left\|\delta\left(\zeta u_{j}\right)\right\|_{L^{p}\left(\Omega \cap B(x, t), \Lambda^{\ell-1}\right)} \leq & C t^{-1}\left\|u_{j}\right\|_{L^{p}\left(\Omega \cap B(x, t), \Lambda^{\ell}\right)} \\
& +\left\|\delta u_{j}\right\|_{L^{p}\left(\Omega \cap B(x, t), \Lambda^{\ell-1}\right)} .
\end{aligned}
$$


Also, the Lipschitz character of $D$ is controlled by that of $\Omega$ and, hence, there is no loss of generality in assuming that $q_{D}=q_{\Omega}$. Thus, we may write

$$
\begin{aligned}
& t^{n\left(\frac{1}{p}-\frac{1}{p^{*}}\right)}\left[\int_{\Omega \cap B(x, t / 2)}\left|u_{j}\right|^{p^{*}} d V\right]^{1 / p^{*}} \leq t^{n\left(\frac{1}{p}-\frac{1}{p^{*}}\right)}\left[\int_{\Omega \cap B(x, t)}\left|\zeta u_{j}\right|^{p^{*}} d V\right]^{1 / p^{*}} \\
& \leq C\left[\int_{\Omega \cap B(x, t)}\left|\zeta u_{j}\right|^{p} d V\right]^{1 / p}+C t\left[\int_{\Omega \cap B(x, t)}\left|d\left(\zeta u_{j}\right)\right|^{p} d V\right]^{1 / p} \\
& +C t\left[\int_{\Omega \cap B(x, t)}\left|\delta\left(\zeta u_{j}\right)\right|^{p} d V\right]^{1 / p} \\
& \leq C\left[\int_{\Omega \cap B(x, t)}\left|u_{j}\right|^{p} d V\right]^{1 / p}+\frac{C}{|\lambda|^{1 / 2}}\left[\int_{\Omega \cap B(x, t)}\left|d u_{j}\right|^{p} d V\right]^{1 / p} \\
& +\frac{C}{|\lambda|^{1 / 2}}\left[\int_{\Omega \cap B(x, t)}\left|\delta u_{j}\right|^{p} d V\right]^{1 / p} \\
& \leq C e^{-c 2^{j}}|\lambda|^{-1}\left[\int_{\Omega}\left|f_{j}\right|^{p} d V\right]^{1 / p},
\end{aligned}
$$

where the last step is based on (5.11). This proves (5.22) with $t$ replaced by $t / 2$. This, however, is easily remedied by carrying out the same program with $t$ replaced by $2 t$ in (5.7), (5.24) and the definition of $D$. Going further, Hölder's inequality and the support condition on $f_{j}$ give

$$
\begin{aligned}
{\left[\int_{\Omega}\left|f_{j}\right|^{p} d V\right]^{1 / p} } & =\left[\int_{\Omega \cap B\left(x, 2^{j+1} t\right)}\left|f_{j}\right|^{p} d V\right]^{1 / p} \\
& \leq C 2^{j n} t^{n\left(\frac{1}{p}-\frac{1}{p^{*}}\right)}\left[\int_{\Omega}\left|f_{j}\right|^{p^{*}} d V\right]^{1 / p^{*}} .
\end{aligned}
$$

In concert with (5.26), this proves a version of (5.23) with $t$ replaced by $t / 2$ though, as before, this aspect is easily fixed.

Step 4. For each $k \in \mathbb{N}$ there exist $C, c>0$ such that

$$
\begin{aligned}
|\lambda|^{1 / 2} & {\left[\int_{\Omega \cap B(x, t)}\left|d u_{j}\right|^{p^{*}} d V\right]^{1 / p^{*}}+|\lambda|^{1 / 2}\left[\int_{\Omega \cap B(x, t)}\left|\delta u_{j}\right|^{p^{*}} d V\right]^{1 / p^{*}} } \\
& \leq C e^{-c 2^{j}} t^{n\left(\frac{1}{p^{*}}-\frac{1}{p}\right)}\left[\int_{\Omega}\left|f_{j}\right|^{p} d V\right]^{1 / p}
\end{aligned}
$$

and

$$
\begin{gathered}
|\lambda|^{1 / 2}\left[\int_{\Omega \cap B(x, t)}\left|d u_{j}\right|^{p^{*}} d V\right]^{1 / p^{*}}+|\lambda|^{1 / 2}\left[\int_{\Omega \cap B(x, t)}\left|\delta u_{j}\right|^{p^{*}} d V\right]^{1 / p^{*}} \\
\leq C e^{-c 2^{j}}\left[\int_{\Omega}\left|f_{j}\right|^{p^{*}} d V\right]^{1 / p^{*}}
\end{gathered}
$$

for each $j \geq 0$.

To justify these inequalities, pick a function $\zeta$ as in (5.24) and invoke (5.19) for $D:=\Omega \cap B(x, t)$ and $w:=\zeta \delta u_{j}$. Note that $\nu \vee\left(\zeta \delta u_{j}\right)=-\zeta \delta_{\partial}\left(\nu \vee u_{j}\right)=0$ on 
$\partial[\Omega \cap B(x, t)]$ and

$$
\begin{gathered}
\left\|\zeta \delta u_{j}\right\|_{L^{p}\left(\Omega \cap B(x, t), \Lambda^{\ell-1}\right)} \leq\left\|\delta u_{j}\right\|_{L^{p}\left(\Omega \cap B(x, t), \Lambda^{\ell-1}\right)}, \\
\left\|d\left(\zeta \delta u_{j}\right)\right\|_{L^{p}\left(\Omega \cap B(x, t), \Lambda^{\ell}\right)} \leq \\
C t^{-1}\left\|\delta u_{j}\right\|_{L^{p}\left(\Omega \cap B(x, t), \Lambda^{\ell-1}\right)} \\
+\left\|d \delta u_{j}\right\|_{L^{p}\left(\Omega \cap B(x, t), \Lambda^{\ell}\right)}, \\
\left\|\delta\left(\zeta \delta u_{j}\right)\right\|_{L^{p}\left(\Omega \cap B(x, t), \Lambda^{\ell-2}\right)} \leq C t^{-1}\left\|\delta u_{j}\right\|_{L^{p}\left(\Omega \cap B(x, t), \Lambda^{\ell-1}\right)} .
\end{gathered}
$$

As before, we may assume that $q_{D}=q_{\Omega}$. Hence, we may estimate

$$
\begin{aligned}
& t^{n\left(\frac{1}{p}-\frac{1}{p^{*}}\right)}\left[\int_{\Omega \cap B(x, t / 2)}\left|\delta u_{j}\right|^{p^{*}} d V\right]^{1 / p^{*}} \leq t^{n\left(\frac{1}{p}-\frac{1}{p^{*}}\right)}\left[\int_{\Omega \cap B(x, t)}\left|\zeta \delta u_{j}\right|^{p^{*}} d V\right]^{1 / p^{*}} \\
& \leq C\left[\int_{\Omega \cap B(x, t)}\left|\zeta \delta u_{j}\right|^{p} d V\right]^{1 / p}+C t\left[\int_{\Omega \cap B(x, t)}\left|d\left(\zeta \delta u_{j}\right)\right|^{p} d V\right]^{1 / p} \\
& \quad+C t\left[\int_{\Omega \cap B(x, t)}\left|\delta\left(\zeta \delta u_{j}\right)\right|^{p} d V\right]^{1 / p} \\
& \leq C\left[\int_{\Omega \cap B(x, t)}\left|\delta u_{j}\right|^{p} d V\right]^{1 / p}+\frac{C}{|\lambda|^{1 / 2}}\left[\int_{\Omega \cap B(x, t)}\left|d \delta u_{j}\right|^{p} d V\right]^{1 / p} \\
& \leq C e^{-c 2^{j}|\lambda|^{-1}\left[\int_{\Omega}\left|f_{j}\right|^{p} d V\right]^{1 / p}}
\end{aligned}
$$

where we have utilized (5.11) and (5.12) in the last step. From this, we readily obtain

$$
|\lambda|^{1 / 2}\left[\int_{\Omega \cap B(x, t)}\left|\delta u_{j}\right|^{p^{*}} d V\right]^{1 / p^{*}} \leq C e^{-c 2^{j}} t^{n\left(\frac{1}{p^{*}}-\frac{1}{p}\right)}\left[\int_{\Omega}\left|f_{j}\right|^{p} d V\right]^{1 / p}
$$

and, in a similar manner,

$$
|\lambda|^{1 / 2}\left[\int_{\Omega \cap B(x, t)}\left|d u_{j}\right|^{p^{*}} d V\right]^{1 / p^{*}} \leq C e^{-c 2^{j}} t^{n\left(\frac{1}{p^{*}}-\frac{1}{p}\right)}\left[\int_{\Omega}\left|f_{j}\right|^{p} d V\right]^{1 / p} .
$$

Together, they prove (5.28). Finally, (5.29) follows from this and (5.27).

Step 5. If $p^{*}<q_{\Omega}$, then for each $k \in \mathbb{N}$ there exist $C, c>0$ such that

$$
\begin{gathered}
{\left[\int_{\Omega \cap B(x, t)}\left|\delta d u_{j}\right|^{p^{*}} d V\right]^{1 / p^{*}}+\left[\int_{\Omega \cap B(x, t)}\left|d \delta u_{j}\right|^{p^{*}} d V\right]^{1 / p^{*}}} \\
\leq C e^{-c 2^{j}} t^{n\left(\frac{1}{p^{*}}-\frac{1}{p}\right)}\left[\int_{\Omega}\left|f_{j}\right|^{p} d V\right]^{1 / p}
\end{gathered}
$$

and

$$
\begin{gathered}
{\left[\int_{\Omega \cap B(x, t)}\left|\delta d u_{j}\right|^{p^{*}} d V\right]^{1 / p^{*}}+\left[\int_{\Omega \cap B(x, t)}\left|d \delta u_{j}\right|^{p^{*}} d V\right]^{1 / p^{*}}} \\
\leq C e^{-c 2^{j}}\left[\int_{\Omega}\left|f_{j}\right|^{p^{*}} d V\right]^{1 / p^{*}}
\end{gathered}
$$

for each $j \geq 3$. 
Assume that $j \geq 3$ and, once again, pick a function $\zeta$ as in (5.24). Thus,

$$
\nu \vee\left(\zeta \delta d u_{j}\right)=-\zeta \delta_{\partial}\left(\nu \vee d u_{j}\right)=0 \text { on } \partial[\Omega \cap B(x, t)] .
$$

Also, since $\zeta f_{j}=0$ for $j \geq 3$,

$$
\zeta \delta d u_{j}=-\zeta \Delta u_{j}-\zeta d \delta u_{j}=-\zeta\left(\lambda u_{j}-f_{j}\right)-\zeta d \delta u_{j}=-\lambda \zeta u_{j}-\zeta d \delta u_{j}
$$

and, hence,

$$
d\left(\zeta \delta d u_{j}\right)=-\lambda \mathcal{O}\left(|\nabla \zeta|\left|u_{j}\right|\right)-\lambda \zeta d u_{j}+\mathcal{O}\left(|\nabla \zeta|\left|d \delta u_{j}\right|\right) .
$$

In particular,

$$
\begin{aligned}
\left\|d\left(\zeta \delta d u_{j}\right)\right\|_{L^{p}\left(\Omega, \Lambda^{\ell+1}\right)} \leq & C|\lambda| t^{-1}\left\|u_{j}\right\|_{L^{p}\left(\Omega, \Lambda^{\ell}\right)}+|\lambda|\left\|d u_{j}\right\|_{L^{p}\left(\Omega, \Lambda^{\ell+1}\right)} \\
& +C t^{-1}\left\|d \delta u_{j}\right\|_{L^{p}\left(\Omega, \Lambda^{\ell}\right)}
\end{aligned}
$$

Since also

$$
\begin{aligned}
& \left\|\zeta \delta d u_{j}\right\|_{L^{p}\left(\Omega \cap B(x, t), \Lambda^{\ell}\right)} \leq\left\|\delta d u_{j}\right\|_{L^{p}\left(\Omega \cap B(x, t), \Lambda^{\ell}\right)}, \\
& \left\|\delta\left(\zeta \delta d u_{j}\right)\right\|_{L^{p}\left(\Omega \cap B(x, t), \Lambda^{\ell}\right)} \leq C t^{-1}\left\|\delta d u_{j}\right\|_{L^{p}\left(\Omega \cap B(x, t), \Lambda^{\ell}\right)},
\end{aligned}
$$

the estimate (5.19) is applicable to $D:=\Omega \cap B(x, t)$ and $w:=\zeta \delta d u_{j}$ (assuming that $q_{D}=q_{\Omega}$, which can be arranged). As a result, we have

$$
\begin{aligned}
& t^{n\left(\frac{1}{p}-\frac{1}{p^{*}}\right)}\left[\int_{\Omega \cap B(x, t / 2)}\left|\delta d u_{j}\right|^{p^{*}} d V\right]^{1 / p^{*}} \leq t^{n\left(\frac{1}{p}-\frac{1}{p^{*}}\right)}\left[\int_{\Omega \cap B(x, t)}\left|\zeta \delta d u_{j}\right|^{p^{*}} d V\right]^{1 / p^{*}} \\
& \quad \leq C\left[\int_{\Omega \cap B(x, t)}\left|\delta d u_{j}\right|^{p} d V\right]^{1 / p}+C|\lambda|\left[\int_{\Omega \cap B(x, t)}\left|u_{j}\right|^{p} d V\right]^{1 / p} \\
& \quad+C|\lambda|^{1 / 2}\left[\int_{\Omega \cap B(x, t)}\left|d u_{j}\right|^{p} d V\right]^{1 / p}+C\left[\int_{\Omega \cap B(x, t)}\left|d \delta u_{j}\right|^{p} d V\right]^{1 / p} \\
& \quad \leq C e^{-c 2^{j}}\left[\int_{\Omega}\left|f_{j}\right|^{p} d V\right]^{1 / p}
\end{aligned}
$$

where the last step utilizes (5.12) and (5.11). In turn, from (5.41), Hölder's inequality and rescaling we readily obtain

$$
\begin{aligned}
{\left[\int_{\Omega \cap B(x, t)}\left|\delta d u_{j}\right|^{p^{*}} d V\right]^{1 / p^{*}} } & \leq C e^{-c 2^{j}} t^{n\left(\frac{1}{p^{*}}-\frac{1}{p}\right)}\left[\int_{\Omega}\left|f_{j}\right|^{p} d V\right]^{1 / p} \\
& \leq C e^{-c 2^{j}}\left[\int_{\Omega}\left|f_{j}\right|^{p^{*}} d V\right]^{1 / p^{*}}
\end{aligned}
$$

Going further, we write $d \delta u_{j}=-\Delta u_{j}-\delta d u_{j}=f_{j}-\lambda u_{j}-\delta d u_{j}$ and, consequently, $d \delta u_{j}=-\lambda u_{j}-\delta d u_{j}$ on $\Omega \cap B(x, t)$ if $j \geq 3$. Hence, based on this, (5.42) and (5.22), 
we may estimate

$$
\begin{aligned}
& {\left[\int_{\Omega \cap B(x, t)}\left|d \delta u_{j}\right|^{p^{*}} d V\right]^{1 / p^{*}}} \\
& \quad \leq|\lambda|\left[\int_{\Omega \cap B(x, t)}\left|u_{j}\right|^{p^{*}} d V\right]^{1 / p^{*}}+\left[\int_{\Omega \cap B(x, t)}\left|\delta d u_{j}\right|^{p^{*}} d V\right]^{1 / p^{*}} \\
& \quad \leq C e^{-c 2^{j}} t^{n\left(\frac{1}{p^{*}}-\frac{1}{p}\right)}\left[\int_{\Omega}\left|f_{j}\right|^{p} d V\right]^{1 / p} \\
& \leq C e^{-c 2^{j}}\left[\int_{\Omega}\left|f_{j}\right|^{p^{*}} d V\right]^{1 / p^{*}} .
\end{aligned}
$$

Clearly, (5.42)-(5.43) prove (5.34)-(5.35).

Step 6. Granted (5.4) - (5.12), for each $q \in\left(p, p^{*}\right]$ there exists $C=C(\partial \Omega, q)>0$ such that

$$
|\lambda|\|u\|_{L^{q}\left(\Omega, \Lambda^{\ell}\right)} \leq C\|f\|_{L^{q}\left(\Omega, \Lambda^{\ell}\right)}
$$

and

$$
|\lambda|^{1 / 2}\|d u\|_{L^{q}\left(\Omega, \Lambda^{\ell+1}\right)}+|\lambda|^{1 / 2}\|\delta u\|_{L^{q}\left(\Omega, \Lambda^{\ell-1}\right)} \leq C\|f\|_{L^{q}\left(\Omega, \Lambda^{\ell}\right)} .
$$

Given $q \in\left(p, p^{*}\right]$, select $\theta \in(0,1]$ such that $1 / q=(1-\theta) / p+\theta / p^{*}$. From (5.11) and (5.23) we then obtain

$$
\begin{aligned}
|\lambda|\left\|u_{j}\right\|_{L^{q}\left(B(x, t) \cap \Omega, \Lambda^{\ell}\right)} & \leq\left[|\lambda|\left\|u_{j}\right\|_{L^{p}\left(B(x, t) \cap \Omega, \Lambda^{\ell}\right)}\right]^{1-\theta}\left[|\lambda|\left\|u_{j}\right\|_{L^{p^{*}}\left(B(x, t) \cap \Omega, \Lambda^{\ell}\right)}\right]^{\theta} \\
& \leq C e^{-c 2^{j}} t^{n\left(\frac{\theta}{p^{*}}-\frac{\theta}{p}\right)}\left\|f_{j}\right\|_{L^{p}\left(\Omega, \Lambda^{\ell}\right)} \\
& =C e^{-c 2^{j}} t^{n\left(\frac{1}{q}-\frac{1}{p}\right)}\left\|f_{j}\right\|_{L^{p}\left(\Omega, \Lambda^{\ell}\right)} .
\end{aligned}
$$

Now, with $f_{E} g d V:=[\text { measure }(E)]^{-1} \int_{E} g d V$, and with $M$ denoting the HardyLittlewood maximal operator, Fubini's Theorem and (5.46) allow us to write

$$
\begin{aligned}
|\lambda|\left[\int_{\Omega}|u|^{q} d V\right]^{1 / q} & \leq C\left[\int_{\Omega}\left(f_{\Omega \cap B(x, t)}|u|^{q} d V\right) d V_{x}\right]^{1 / q} \\
& =C|\lambda|\left\{\int_{\Omega}\left[\left(f_{\Omega \cap B(x, t)}|u|^{q} d V\right)^{1 / q}\right]^{q} d V_{x}\right\}^{1 / q} \\
& \left.\left.\left.\leq C|\lambda|\left\{\int_{\Omega}\left[\sum_{j=0}^{\infty}\left(f_{\Omega \cap B(x, t)}\left|u_{j}\right|^{q} d V\right)^{1 / q}\right]^{q} d V_{x}\right\}^{1 / q}|f|^{p} d V\right)^{1 / p}\right]^{q} d V_{x}\right\}^{1 / q} \\
& \leq C\left\{\int _ { \Omega } \left[\sum _ { j = 0 } ^ { \infty } C e ^ { - c 2 ^ { j } } 2 ^ { j n / p } \left(f_{\Omega \cap B\left(x, 2^{j} t\right)}\right.\right.\right. \\
& \leq C\left(\sum_{j=0}^{\infty} e^{-c 2^{j}} 2^{j n / p}\right)\left\|M\left(|f|^{p}\right)\right\|_{L^{q / p}(\Omega)}^{1 / p} \\
& \leq C\|f\|_{L^{q}(\Omega)} .
\end{aligned}
$$


This proves (5.44). The estimate (5.45) is then justified in a similar manner, by relying on (5.11) and (5.29).

Step 7. The estimate (5.35) also holds if $0 \leq j \leq 3$.

It suffices to show that there exists $C=C(\partial \Omega, p)>0$ such that

$$
\left\|\delta d u_{j}\right\|_{L^{p^{*}}\left(\Omega, \Lambda^{\ell}\right)}+\left\|d \delta u_{j}\right\|_{L^{p^{*}}\left(\Omega, \Lambda^{\ell}\right)} \leq C\left\|f_{j}\right\|_{L^{p^{*}}\left(\Omega, \Lambda^{\ell}\right)}
$$

for each $j \geq 0$. To this end, we first note that the conclusion in Step 6 (with $q=p^{*}$ ) applied to $u_{j}, f_{j}$ in place of $u, f$ yields

$$
|\lambda|\left\|u_{j}\right\|_{L^{p^{*}}\left(\Omega, \Lambda^{\ell}\right)} \leq C\left\|f_{j}\right\|_{L^{p^{*}}\left(\Omega, \Lambda^{\ell}\right)}, \quad \forall j \geq 0 .
$$

Next, recall that $u_{j} \in \operatorname{Dom}\left(B_{2}\right)$ and $(\lambda I-\Delta) u_{j}=f_{j} \in C_{0}^{\infty}\left(\Omega, \Lambda^{\ell}\right) \hookrightarrow L^{p^{*}}\left(\Omega, \Lambda^{\ell}\right)$. Since our current assumptions imply $2<p^{*}<q_{\Omega}$, Proposition 4.1 guarantees that $u_{j} \in \operatorname{Dom}\left(B_{p^{*}}\right)$. Consequently, (4.11) and (5.49) allow us to write

$$
\begin{aligned}
\left\|d \delta u_{j}\right\|_{L^{p^{*}}\left(\Omega, \Lambda^{\ell}\right)}+\left\|\delta d u_{j}\right\|_{L^{p^{*}}\left(\Omega, \Lambda^{\ell}\right)} \leq C\left\|\Delta u_{j}\right\|_{L^{p^{*}}\left(\Omega, \Lambda^{\ell}\right)} & \\
& =C\left\|\lambda u_{j}-f_{j}\right\|_{L^{p^{*}}\left(\Omega, \Lambda^{\ell}\right)} \leq C \mid \lambda\|\| u_{j}\left\|_{L^{p^{*}}\left(\Omega, \Lambda^{\ell}\right)}+C\right\| f_{j} \|_{L^{p^{*}}\left(\Omega, \Lambda^{\ell}\right)} \\
& \leq C\left\|f_{j}\right\|_{L^{p^{*}}\left(\Omega, \Lambda^{\ell}\right)}, \quad \forall j \geq 0,
\end{aligned}
$$

for some finite $C=C(\partial \Omega, p)>0$. Thus, (5.48) is proved.

Step 8. Proof of (5.13), (5.15).

Note that (5.13) is a consequence of (5.23) and (5.29). Finally, (5.35) takes care of the case $j \geq 3$ of (5.15), whereas the case $0 \leq j \leq 3$ is contained in Step 7 .

This finishes the proof of Lemma 5.1

\section{Resolvent Estimates}

In this section we shall make use of Lemma 5.1 in order to prove resolvent estimates for the Hodge Laplacian. To state our main result in this regard, recall the definition of the sector $\Sigma_{\theta}$ from (5.1). Also, given a Lipschitz domain $\Omega \subset \mathcal{M}$, $\operatorname{dim} \mathcal{M}=n$, recall the critical exponents $p_{\Omega}, q_{\Omega}$ from (3.1), and set

$$
q_{\Omega}^{*}:=\frac{n q_{\Omega}}{n-1}, \quad\left(q_{\Omega}^{*}\right)^{\prime}:=\left(1-\frac{1}{q_{\Omega}^{*}}\right)^{-1} .
$$

Theorem 6.1. Let $\Omega \subset \mathcal{M}$ be a Lipschitz domain, and fix $\ell \in\{0,1, \ldots, n\}$ and $\theta \in(0, \pi)$. Then for each $\lambda \in \Sigma_{\theta}$ and each

$$
p \in\left(p_{\Omega}, q_{\Omega}\right)
$$

the operator

$$
\lambda I+B_{p}: \operatorname{Dom}\left(B_{p}\right) \subset L^{p}\left(\Omega, \Lambda^{\ell}\right) \longrightarrow L^{p}\left(\Omega, \Lambda^{\ell}\right)
$$

has a bounded inverse. In addition, there exists $C=C(\partial \Omega, \theta, p)>0$ such that

$$
\begin{aligned}
& \left\|\left(\lambda I+B_{p}\right)^{-1} f\right\|_{L^{p}\left(\Omega, \Lambda^{\ell}\right)} \leq C|\lambda|^{-1}\|f\|_{L^{p}\left(\Omega, \Lambda^{\ell}\right)} \\
& \left\|d\left(\lambda I+B_{p}\right)^{-1} f\right\|_{L^{p}\left(\Omega, \Lambda^{\ell-1}\right)}+\left\|\delta\left(\lambda I+B_{p}\right)^{-1} f\right\|_{L^{p}\left(\Omega, \Lambda^{\ell+1}\right)} \\
& \leq C|\lambda|^{-1 / 2}\|f\|_{L^{p}\left(\Omega, \Lambda^{\ell}\right)}, \\
& \left\|d \delta\left(\lambda I+B_{p}\right)^{-1} f\right\|_{L^{p}\left(\Omega, \Lambda^{\ell}\right)}+\left\|\delta d\left(\lambda I+B_{p}\right)^{-1} f\right\|_{L^{p}\left(\Omega, \Lambda^{\ell}\right)} \leq C\|f\|_{L^{p}\left(\Omega, \Lambda^{\ell}\right)},
\end{aligned}
$$

for any $\lambda \in \Sigma_{\theta}$ and any $f \in L^{p}\left(\Omega, \Lambda^{\ell}\right)$. 
Moreover, for each

$$
p \in\left(\left(q_{\Omega}^{*}\right)^{\prime}, q_{\Omega}^{*}\right)
$$

the mapping $\left(\lambda I+B_{2}\right)^{-1}: L^{2}\left(\Omega, \Lambda^{\ell}\right) \rightarrow L^{2}\left(\Omega, \Lambda^{\ell}\right)$ extends to a bounded, linear operator from $L^{p}\left(\Omega, \Lambda^{\ell}\right)$ into itself which continues to satisfy (6.4) and (6.5).

Proof. Consider first the case when $p \in\left(2, q_{\Omega}\right)$. In this scenario, for an arbitrary $\lambda \in \Sigma_{\theta}$, the fact that the operator (6.3) is one-to-one follows trivially from the corresponding statement for $p=2$ (dealt with in $\S 3$ ). To see that this operator is also onto, let $f \in L^{p}\left(\Omega, \Lambda^{\ell}\right) \hookrightarrow L^{p}\left(\Omega, \Lambda^{\ell}\right)$ and consider $u:=\left(\lambda I+B_{2}\right)^{-1} f \in$ $\operatorname{Dom}\left(B_{2}\right)$. Thanks to Proposition 4.1, we have that $u \in \operatorname{Dom}\left(B_{p}\right)$ and $\left(\lambda I+B_{p}\right) u=$ $f$, which proves that the operator (6.3) is indeed onto.

Turning our attention to (6.4)-(6.5), we note that it suffices to prove these estimates for an arbitrary $f \in C_{0}^{\infty}\left(\Omega, \Lambda^{\ell}\right)$. With the notation and conventions introduced in $\S 5$, these are going to be consequences of (5.44) and (5.45), provided we show that the index $q$ appearing there can be chosen arbitrarily in $\left(2, q_{\Omega}\right)$. In turn, by virtue of the inductive bootstrap argument in Lemma 5.1, this latter condition will hold as soon as we prove that (5.11)-(5.12) are valid for the choice $p=2$.

The argument utilizes a Caccioppoli-type inequality along with a trick we have learned from [2]. More concretely, we start by pairing both sides of (5.17) with $\bar{u}_{j}$ in the $L^{2}$-sense. After integrating by parts, we eventually obtain

$$
\lambda \int_{\Omega}\left|u_{j}\right|^{2} d V+\int_{\Omega}\left|d u_{j}\right|^{2} d V+\int_{\Omega}\left|\delta u_{j}\right|^{2} d V=\int_{\Omega}\left\langle f_{j}, \bar{u}_{j}\right\rangle d V .
$$

From this we may further deduce, based on (5.2) and the Cauchy-Schwarz inequality, that

$$
|\lambda|\left\|u_{j}\right\|_{L^{2}\left(\Omega, \Lambda^{\ell}\right)}+|\lambda|^{1 / 2}\left\|d u_{j}\right\|_{L^{2}\left(\Omega, \Lambda^{\ell+1}\right)}+|\lambda|^{1 / 2}\left\|\delta u_{j}\right\|_{L^{2}\left(\Omega, \Lambda^{\ell-1}\right)} \leq C\left\|f_{j}\right\|_{L^{2}\left(\Omega, \Lambda^{\ell}\right)} .
$$

Next, with $t$ retaining the same significance as before, i.e. $t:=|\lambda|^{-1 / 2}$, pick a new family of functions $\left\{\xi_{j}\right\}_{j \geq 3}$ such that

$$
\xi_{j} \in C_{o}^{\infty}\left(B\left(x, 2^{j-2} t\right)\right), \quad \text { for each } j \geq 3 .
$$

Taking the $L^{2}$-pairing of $\xi_{j}^{2} \bar{u}_{j}$ with both sides of (5.17) and keeping in mind that $\xi_{j} \eta_{j}=0$ for each $j \geq 3$ we may write, based on integrations by parts that

$$
\begin{array}{r}
\lambda \int_{\Omega} \xi_{j}^{2}\left|u_{j}\right|^{2} d V+\int_{\Omega} \xi_{j}^{2}\left|d u_{j}\right|^{2} d V+\int_{\Omega} \xi_{j}^{2}\left|\delta u_{j}\right|^{2} d V \\
=\int_{\Omega} \mathcal{O}\left(\left|\nabla \xi_{j}\right|\left|u_{j}\right|\left[\left|\xi_{j}\right|\left|d u_{j}\right|+\left|\xi_{j}\right|\left|\delta u_{j}\right|\right]\right) d V .
\end{array}
$$

From this and (5.2) we then obtain that

$$
\begin{aligned}
& |\lambda| \int_{\Omega} \xi_{j}^{2}\left|u_{j}\right|^{2} d V+\int_{\Omega} \xi_{j}^{2}\left|d u_{j}\right|^{2} d V+\int_{\Omega} \xi_{j}^{2}\left|\delta u_{j}\right|^{2} d V \\
& \quad \leq C \int_{\Omega}\left|\nabla \xi_{j}\right|\left|u_{j}\right|\left[\left|\xi_{j}\right|\left|d u_{j}\right|+\left|\xi_{j}\right|\left|\delta u_{j}\right|\right] d V, \quad \text { for } j \geq 3,
\end{aligned}
$$


which, via Cauchy-Schwarz's inequality and a standard trick that allows us to absorb like-terms with small coefficients in the left-hand side, further gives

$$
\begin{gathered}
|\lambda| \int_{\Omega} \xi_{j}^{2}\left|u_{j}\right|^{2} d V+\int_{\Omega} \xi_{j}^{2}\left|d u_{j}\right|^{2} d V+\int_{\Omega} \xi_{j}^{2}\left|\delta u_{j}\right|^{2} d V \\
\leq C \int_{\Omega}\left|\nabla \xi_{j}\right|^{2}\left|u_{j}\right|^{2} d V, \text { for each } j \geq 3 .
\end{gathered}
$$

In particular,

$$
\begin{aligned}
& \int_{\Omega} \xi_{j}^{2}\left|d u_{j}\right|^{2} d V+\int_{\Omega} \xi_{j}^{2}\left|\delta u_{j}\right|^{2} d V \leq C \int_{\Omega}\left|\nabla \xi_{j}\right|^{2}\left|u_{j}\right|^{2} d V \text { and } \\
& |\lambda| \int_{\Omega} \xi_{j}^{2}\left|u_{j}\right|^{2} d V \leq C \int_{\Omega}\left|\nabla \xi_{j}\right|^{2}\left|u_{j}\right|^{2} d V, \quad \forall j \geq 3 .
\end{aligned}
$$

Much as in 2], we now replace the cutoff function $\xi_{j}$ in (6.14) by $e^{\alpha_{j} \xi_{j}}-1$ (which once again obeys (6.10) $)$, where

$$
\alpha_{j}:=\frac{\sqrt{|\lambda|}}{2 C\left\|\nabla \xi_{j}\right\|_{L^{\infty}}}, \quad j \geq 3
$$

In a first stage, this yields

$$
\int_{\Omega}\left|u_{j}\right|^{2}\left|e^{\alpha_{j} \xi_{j}}-1\right|^{2} d V \leq \frac{1}{4} \int_{\Omega}\left|u_{j}\right|^{2}\left|e^{\alpha_{j} \xi_{j}}\right|^{2} d V, \quad \forall j \geq 3,
$$

then, further,

$$
\int_{\Omega}\left|u_{j}\right|^{2}\left|e^{\alpha_{j} \xi_{j}}\right|^{2} d V \leq 4 \int_{\Omega}\left|u_{j}\right|^{2} d V, \quad \forall j \geq 3
$$

in view of the generic, elementary implication $\|f-g\| \leq \frac{1}{2}\|f\| \Rightarrow\|f\| \leq 2\|g\|$. If we now assume that the original cutoff functions $\left\{\xi_{j}\right\}_{j}$ also satisfy

$$
\xi_{j} \equiv 1 \text { on } B\left(x, 2^{j-3} t\right), \quad 0 \leq \xi_{j} \leq 1, \quad\left|\nabla \xi_{j}\right| \leq \frac{C}{2^{j} t}, \quad \text { for each } j \geq 3,
$$

it follows from (6.15) that $\alpha_{j} \geq c 2^{j}$, and from (6.17) that

$$
\left|e^{\alpha_{j}}\right|^{2} \int_{\Omega \cap B\left(x, 2^{j-3} t\right)}\left|u_{j}\right|^{2} d V \leq 4 \int_{\Omega}\left|u_{j}\right|^{2} d V, \quad \forall j \geq 3 .
$$

In concert with (5.11), the above analysis shows that there exist $C, c>0$ such that

$$
\begin{aligned}
|\lambda| \int_{\Omega \cap B\left(x, 2^{j-3} t\right)}\left|u_{j}\right|^{2} d V & \leq C|\lambda| e^{-c 2^{j}} \int_{\Omega}\left|u_{j}\right|^{2} d V, \\
& \leq C \frac{e^{-c 2^{j}}}{|\lambda|} \int_{\Omega}\left|f_{j}\right|^{2} d V, \quad \forall j \geq 3 .
\end{aligned}
$$

All in all, from (6.9) with $0 \leq j \leq 3$ and (6.20) with $j>3$, we obtain

$$
|\lambda| \int_{\Omega \cap B(x, t)}\left|u_{j}\right|^{2} d V \leq C \frac{e^{-c 2^{j}}}{|\lambda|} \int_{\Omega}\left|f_{j}\right|^{2} d V, \quad \forall j \geq 0 .
$$

Furthermore, (6.13), (6.9), (6.18) and (6.20) also imply that, for some $C, c>0$,

$$
\int_{\Omega}\left[\left|d u_{j}\right|^{2}+\left|\delta u_{j}\right|^{2}\right] d V \leq C \frac{e^{-c 2^{j}}}{|\lambda|} \int_{\Omega}\left|f_{j}\right|^{2} d V, \quad \forall j \geq 0 .
$$


There remains to prove the $p=2$ version of (5.12), a task to which we now turn. In fact, we aim at showing that

$$
\int_{B(x, t / 2) \cap \Omega}\left\{\left|d \delta u_{j}\right|^{2}+\left|\delta d u_{j}\right|^{2}\right\} d V \leq C e^{-c 2^{j}} \int_{\Omega}\left|f_{j}\right|^{2} d V, \quad \forall j \geq 0,
$$

which corresponds to (5.12) written for $p=2$ and $t / 2$ in place of $t$ (the latter condition being just a minor technicality, easily addressed via rescaling). In turn, if $\zeta$ is as in (5.24), (6.23) will be a simple consequence of the estimate

$$
\int_{B(x, t) \cap \Omega}\left\{\left|d\left(\zeta \delta u_{j}\right)\right|^{2}+\left|\delta\left(\zeta d u_{j}\right)\right|^{2}\right\} d V \leq C e^{-c 2^{j}} \int_{\Omega}\left|f_{j}\right|^{2} d V,
$$

which, so we claim, is valid for each $j \geq 0$. In order to justify (6.24), we shall first establish the estimate

$$
\int_{B(x, t) \cap \Omega}\left|\zeta \Delta u_{j}\right|^{2} d V \leq C e^{-c 2^{j}} \int_{\Omega}\left|f_{j}\right|^{2} d V, \quad \forall j \geq 0 .
$$

To prove this, we first note that since $\Delta u_{j}=\lambda u_{j}-f_{j}$ in $\Omega$ for every $j$, then

$$
\left\|\Delta u_{j}\right\|_{L^{2}\left(\Omega, \Lambda^{\ell}\right)} \leq|\lambda|\left\|u_{j}\right\|_{L^{2}\left(\Omega, \Lambda^{\ell}\right)}+\left\|f_{j}\right\|_{L^{2}\left(\Omega, \Lambda^{\ell}\right)} \leq C\left\|f_{j}\right\|_{L^{2}\left(\Omega, \Lambda^{\ell}\right)}
$$

by (6.9). As this implies (6.25) for small $j$ 's, we can assume for the remainder of the proof that $j \geq 3$. In particular, $f_{j} \equiv 0$ on $B(x, t)$. Next, multiply by $\zeta$ both sides of the equality $\Delta u_{j}=\lambda u_{j}-f_{j}$ to get $\zeta \Delta u_{j}=\lambda \zeta u_{j}$ and write

$$
\begin{aligned}
\int_{B(x, t) \cap \Omega}\left|\zeta \Delta u_{j}\right|^{2} d V & =|\lambda|^{2} \int_{B(x, t) \cap \Omega}\left|\zeta u_{j}\right|^{2} d V \\
& \leq|\lambda|^{2} \int_{B(x, t) \cap \Omega}\left|u_{j}\right|^{2} d V \leq C e^{-c 2^{j}} \int_{\Omega}\left|f_{j}\right|^{2} d V
\end{aligned}
$$

where in the last step we have used (6.20). This finishes the proof of (6.25).

To continue, write

$$
-\zeta \Delta u_{j}=d\left(\zeta \delta u_{j}\right)+\delta\left(\zeta d u_{j}\right)+\mathcal{O}\left(|\nabla \zeta|\left[\left|d u_{j}\right|+\left|\delta u_{j}\right|\right]\right)
$$

so that

$$
\begin{aligned}
& \int_{B(x, t) \cap \Omega}\left|d\left(\zeta \delta u_{j}\right)+\delta\left(\zeta d u_{j}\right)\right|^{2} d V \\
& \quad=\int_{B(x, t) \cap \Omega}\left|\zeta \Delta u_{j}\right|^{2} d V+\int_{B(x, t) \cap \Omega} \mathcal{O}\left(|\nabla \zeta|^{2}\left[\left|d u_{j}\right|^{2}+\left|\delta u_{j}\right|^{2}\right]\right) d V \\
& \leq C e^{-c 2^{j}} \int_{\Omega}\left|f_{j}\right|^{2} d V+|\lambda| \int_{B(x, t) \cap \Omega}\left[\left|d u_{j}\right|^{2}+\left|\delta u_{j}\right|^{2}\right] d V \\
& \leq C e^{-c 2^{j}} \int_{\Omega}\left|f_{j}\right|^{2} d V
\end{aligned}
$$

by (6.25), (5.24) and (6.22). On the other hand,

$$
\left|d\left(\zeta \delta u_{j}\right)\right|^{2}+\left|\delta\left(\zeta d u_{j}\right)\right|^{2}=\left|d\left(\zeta \delta u_{j}\right)+\delta\left(\zeta d u_{j}\right)\right|^{2}-2 \operatorname{Re}\left\langle d\left(\zeta \delta u_{j}\right), \delta\left(\zeta d u_{j}\right)\right\rangle
$$

and, via an integration by parts,

$$
\int_{B(x, t) \cap \Omega}\left\langle d\left(\zeta \delta u_{j}\right), \delta\left(\zeta d u_{j}\right)\right\rangle d V=0
$$


since $d^{2}=0$ and $\nu \vee\left(\zeta d u_{j}\right)=\zeta\left(\nu \vee d u_{j}\right)=0$ on $\partial[\Omega \cap B(x, t)]$. Thus, all in all, (6.24) is a consequence of (6.30), (6.29) and (6.31), and this finishes the proof of (5.12) when $p=2$. In turn, as explained earlier, this concludes the proof of (6.4)-(6.5) in the case when $2<p<q_{\Omega}$.

As regards the estimate (6.6), we may invoke Proposition 4.2. (6.4) and the fact that $\Delta\left(\lambda I+B_{p}\right)^{-1} f=\lambda\left(\lambda I+B_{p}\right)^{-1} f+f$ in order to justify it in the case when $2<p<q_{\Omega}$. Now, the case when $p_{\Omega}<p<2$ follows from what we have proved so far and duality; cf. Proposition 3.3 Since the case $p=2$ is implicit in the above analysis, this finishes the proof of (6.4)-(6.6). Finally, the last claim in the statement of the theorem is a consequence of what we have proved up to this point and Lemma 5.1. This completes the proof of Theorem 6.1.

\section{AnAlytic Semigroups}

We start with the case of the Hodge Laplacian, for which we have:

Theorem 7.1. If $\Omega \subset \mathcal{M}$ is a Lipschitz domain and $0 \leq \ell \leq n$, then the operator $-B_{p}$ generates an analytic semigroup in $L^{p}\left(\Omega, \Lambda^{\ell}\right)$ for each $p \in\left(p_{\Omega}, q_{\Omega}\right)$. More specifically, for each $\theta \in(0, \pi / 2)$ there exists an analytic map

$$
T_{p}: \Sigma_{\theta} \longrightarrow \mathcal{L}\left(L^{p}\left(\Omega, \Lambda^{\ell}\right), L^{p}\left(\Omega, \Lambda^{\ell}\right)\right)
$$

such that the following hold:

$$
\begin{aligned}
& \lim _{\substack{z \rightarrow 0 \\
z \in \Sigma_{\theta}}} T_{p}(z) f=f \text { in } L^{p}\left(\Omega, \Lambda^{\ell}\right), \forall f \in L^{p}\left(\Omega, \Lambda^{\ell}\right), \\
& T_{p}\left(z_{1}+z_{2}\right)=T_{p}\left(z_{1}\right) T_{p}\left(z_{2}\right), \quad \forall z_{1}, z_{2} \in \Sigma_{\theta}, \\
& \operatorname{Dom}\left(B_{p}\right)=\left\{u \in L^{p}\left(\Omega, \Lambda^{\ell}\right): \lim _{t \rightarrow 0^{+}} \frac{T_{p}(t) u-u}{t} \text { exists in } L^{p}\left(\Omega, \Lambda^{\ell}\right)\right\}, \\
& -B_{p} u=\lim _{t \rightarrow 0^{+}} \frac{T_{p}(t) u-u}{t} \text { for each } u \in \operatorname{Dom}\left(B_{p}\right) .
\end{aligned}
$$

Furthermore, whenever $p \in\left(\left(q_{\Omega}^{*}\right)^{\prime}, q_{\Omega}^{*}\right)$, the semigroup (7.1) further extends to a mapping $\widetilde{T}_{p}: \Sigma_{\theta} \longrightarrow \mathcal{L}\left(L^{p}\left(\Omega, \Lambda^{\ell}\right), L^{p}\left(\Omega, \Lambda^{\ell}\right)\right)$ which continues to satisfy (17.3). For this range of $p$ 's, this extension satisfies

$$
\left(\widetilde{T}_{p}\right)^{*}=\widetilde{T}_{p^{\prime}}, \quad 1 / p+1 / p^{\prime}=1 .
$$

Proof. The first part follows from Theorem 6.1 and the standard theory; cf., e.g., Theorem 5.2 on p. 61 of 23 and the comments preceding it. The second part is a consequence of this and the last claim in the statement of Theorem 6.1.

As is customary, we shall set

$$
e^{-t B_{p}}:=T_{p}(t), \quad t>0 .
$$

Corollary 7.2. Under the hypotheses of Theorem 7.1, for each $t>0$ and $p \in$ $\left(p_{\Omega}, q_{\Omega}\right)$ one has

$$
\mathbb{P}_{p} e^{-t B_{p}}=e^{-t B_{p}} \mathbb{P}_{p}, \quad \mathbb{Q}_{p} e^{-t B_{p}}=e^{-t B_{p}} \mathbb{Q}_{p}
$$

Proof. This is an immediate consequence of Theorem 7.1 Lemma 3.7 and (ii) in Lemma 3.13. 
Theorem 7.3. For a Lipschitz domain $\Omega \subset \mathcal{M}, 0 \leq \ell \leq n$, and $1<p<\infty$, let $A_{p}$ denote the Stokes operator as introduced in $\S 3.2$. Then $-A_{p}$ generates an analytic semigroup $\left(e^{-t A_{p}}\right)_{t>0}$ on the space $X^{p}\left(\Omega, \Lambda^{\ell}\right)$ provided $p \in\left(p_{\Omega}, q_{\Omega}\right)$.

Furthermore, for each $t>0$,

$$
\left(e^{-t A_{p}}\right)^{*}=e^{-t A_{p^{\prime}}}, \quad 1 / p+1 / p^{\prime}=1,
$$

and

$$
\mathbb{P}_{p} e^{-t B_{p}}=e^{-t A_{p}} \mathbb{P}_{p} \quad \text { on } \quad L^{p}\left(\Omega, \Lambda^{\ell}\right)
$$

whenever $p \in\left(p_{\Omega}, q_{\Omega}\right)$.

Proof. Fix some $\theta \in(0, \pi / 2)$ and assume that $p \in\left(p_{\Omega}, q_{\Omega}\right)$. Using Lemma 3.10 and Theorem 6.1, for each $f \in X^{p}\left(\Omega, \Lambda^{\ell}\right)$ we may then write

$$
\begin{aligned}
\left\|\left(\lambda I+A_{p}\right)^{-1} f\right\|_{L^{p}\left(\Omega, \Lambda^{\ell}\right)} & =\left\|\left(\lambda I+A_{p}\right)^{-1} \mathbb{P}_{p} f\right\|_{L^{p}\left(\Omega, \Lambda^{\ell}\right)} \\
& =\left\|\mathbb{P}_{p}\left(\lambda I+B_{p}\right)^{-1} f\right\|_{L^{p}\left(\Omega, \Lambda^{\ell}\right)} \\
& \leq C|\lambda|^{-1}\|f\|_{L^{p}\left(\Omega, \Lambda^{\ell}\right)},
\end{aligned}
$$

uniformly for $\lambda \in \Sigma_{\theta}$. Consequently, $-A_{p}$ generates an analytic semigroup on $X^{p}\left(\Omega, \Lambda^{\ell}\right)$ whenever $p \in\left(p_{\Omega}, q_{\Omega}\right)$.

Finally, (7.9) and (7.10) follow readily from this, Lemma 3.8 and Lemma 3.14 . completing the proof of the theorem.

In a similar fashion, one can prove the following.

Theorem 7.4. If $\Omega \subset \mathcal{M}$ is a Lipschitz domain, $0 \leq \ell \leq n, 1<p<\infty$, and if $C_{p}$ denotes the Maxwell operator, then $-C_{p}$ generates an analytic semigroup $\left(e^{-t C_{p}}\right)_{t>0}$ on the space $Z^{p}\left(\Omega, \Lambda^{\ell}\right)$ whenever $p \in\left(p_{\Omega}, q_{\Omega}\right)$. Moreover, for each $t>0$,

$$
\left(e^{-t C_{p}}\right)^{*}=e^{-t C_{p^{\prime}}}, \quad 1 / p+1 / p^{\prime}=1,
$$

and

$$
\mathbb{Q}_{p} e^{-t B_{p}}=e^{-t C_{p}} \mathbb{Q}_{p} \text { on } L^{p}\left(\Omega, \Lambda^{\ell}\right),
$$

for each $p \in\left(p_{\Omega}, q_{\Omega}\right)$.

Theorem 7.5. Fix $0 \leq \ell \leq n$ and suppose that $\Omega \subset \mathcal{M}$ is a Lipschitz domain for which $b_{\ell}=0$. Then for each $p \in\left(p_{\Omega}, q_{\Omega}\right)$, the analytic semigroups generated by the operators $-A_{p},-B_{p}$ and $-C_{p}$, respectively, on $X^{p}\left(\Omega, \Lambda^{\ell}\right), L^{p}\left(\Omega, \Lambda^{\ell}\right)$ and $Z^{p}\left(\Omega, \Lambda^{\ell}\right)$ are bounded.

Proof. This follows from Theorems 7.1, 7.3 and 7.4, given that under the current topological assumptions the operators $A_{p}, B_{p}$ and $C_{p}$ are invertible.

In closing, we would like to point out that, as an obvious corollary of what we have proved so far, similar results are valid for the Hodge duals of the operators $A_{p}, B_{p}$ and $C_{p}$ (i.e., for $* A_{p} *, * B_{p} *$ and $* C_{p} *$ ). For example, corresponding to the Hodge dual of $B_{p},-\Delta$, defined as an unbounded operator on $L^{p}\left(\Omega, \Lambda^{\ell}\right)$ with domain

$\left\{u \in \mathcal{D}_{\ell}^{p}(\Omega ; d) \cap \mathcal{D}_{\ell}^{p}(\Omega ; \delta): d u \in \mathcal{D}_{\ell+1}^{p}(\Omega ; \delta), \delta u \in \mathcal{D}_{\ell-1}^{p}(\Omega ; d), \nu \wedge u=0, \nu \wedge \delta u=0\right\}$, 
generates an analytic semigroup whenever $p_{\Omega}<p<q_{\Omega}$. We leave the details for the remaining operators to the interested reader.

\section{ACKNOWLEDGMENTS}

This work was completed during several visits at the Université Aix-Marseille 3, France, and the University of Missouri-Columbia, USA. The authors gratefully acknowledge the hospitality of these institutions. The authors are also indebted to the referee for his/her careful reading of the paper and useful suggestions.

\section{REFERENCES}

[1] S. Agmon, On the eigenfunctions and on the eigenvalues of general elliptic boundary value problems, Comm. Pure Appl. Math., 15 (1962), 119-147. MR0147774(26:5288)

[2] P. Auscher, S. Hofmann, M. Lacey, A. McIntosh and P. Tchamitchian, The solution of the Kato square root problem for second order elliptic operators on $\mathbb{R}^{n}$, Ann. of Math. (2), 156 (2002), no. 2, 633-654. MR1933726 (2004c:47096c)

[3] T. Akiyama, H. Kasai, Y. Shibata and M. Tsutsumi, The $L^{p}$ approach to the Laplace system with some boundary conditions and applying to the parabolic system, unpublished lecture notes, 1999.

[4] T. Akiyama, H. Kasai, Y. Shibata and M. Tsutsumi, On a resolvent estimate of a system of Laplace operators with perfect wall condition, Funkcial. Ekvac., 47 (2004), no. 3, 361-394. MR2126323 (2005m:35076)

[5] V. Barbu, T. Havarneanu, C. Popa and S.S. Sritharan, Exact controllability for the magnetohydrodynamic equations, Comm. Pure Appl. Math., 56 (2003), no. 6, 732-783. MR1959739 (2004b:93012)

[6] P.E. Conner, The Neumann's problem for differential forms on Riemannian manifolds, Mem. Amer. Math. Soc., No. 20, 1956. MR0078467 (17:1197e)

[7] T.G. Cowling, Magnetohydrodynamics, Interscience Tracts on Physics and Astronomy, No. 4, Interscience Publishers, Inc., New York, London, 1957. MR0098556 (20:5013)

[8] R. Dautray and J.-L.Lions, Analyse Mathématique et Calcul Numérique pour les Sciences et les Techniques, Vol. 8, Évolution: Semi-groupe, Variationnel. Masson, Paris, 1988. MR1016605 (90m:00006b)

[9] P. Deuring, $L^{p}$-theory for the Stokes system in $3 D$ domains with conical boundary points, Indiana Univ. Math. J., 47 (1998), 11-47. MR1631608(99j:35162)

[10] E.B. Fabes, J.E. Lewis and N.M.Rivière, Boundary value problems for the Navier-Stokes equations, Amer. J. Math., 99 (1977), no. 3, 626-668. MR0460928 (57:919)

[11] E. Fabes, O. Mendez and M. Mitrea, Potential operators on Besov spaces and the Poisson equation with Dirichlet and Neumann boundary conditions on Lipschitz domains, J. Funct. Anal., 159 (1998), no. 2, 323-368. MR1658089 (99j:35036)

[12] Y. Giga, Analyticity of the semigroup generated by the Stokes operator in $L_{r}$ spaces, Math. Z., 178 (1981), no. 3, 297-329. MR635201 (83e:47028)

[13] D. Jerison and C. Kenig, The inhomogeneous Dirichlet problem in Lipschitz domains, J. Funct. Anal., 130 (1995), 161-219. MR.1331981 (96b:35042)

[14] A. Jonsson and H. Wallin, Function Spaces on Subsets of $\mathbb{R}^{n}$, Harwood Academic, New York, 1984. MR820626 (87f:46056)

[15] T.Kato, Perturbation Theory for Linear Operators, Springer-Verlag, Berlin, 1995. MR.1335452 (96a:47025)

[16] L.D. Landau and E.M. Lifshitz, Electrodynamics of Continuous Media, Pergamon Press, New York; Addison-Wesley Publishing Co., Inc., Reading, Mass., 1960. MR.0121049 (22:11796)

[17] T. Miyakawa, The $L^{p}$ approach to the Navier-Stokes equations with the Neumann boundary condition, Hiroshima Math. J., 10 (1980), no. 3, 517-537. MR.594132 (82c:35067)

[18] D. Mitrea, M. Mitrea and M. Taylor, Layer potentials, the Hodge Laplacian, and global boundary problems in nonsmooth Riemannian manifolds, Memoirs of the Amer. Math. Soc., Vol. 150, No. 713, 2001. MR1809655 (2002g:58026)

[19] D. Mitrea and M. Mitrea, Finite energy solutions of Maxwell's equations and constructive Hodge decompositions on nonsmooth Riemannian manifolds, J. Funct. Anal., 190 (2002), 339-417. MR1899489 (2003m:58002) 
[20] M. Mitrea, Sharp Hodge decompositions, Maxwell's equations, and vector Poisson problems on nonsmooth, three-dimensional Riemannian manifolds, Duke Math. J., 125 (2004), 467547. MR 2166752 (2007g:35246)

[21] M. Mitrea and S. Monniaux, The nonlinear Hodge-Navier-Stokes equations in Lipschitz domains, to appear in the Journal of Differential and Integral Equations, (2008).

[22] M. Mitrea, M. Taylor and A. Vasy, Lipschitz domains, domains with corners, and the Hodge Laplacian, Communications in PDE, 30 (2005), no. 10, 1445-1462. MR2182300|(2006h:58029)

[23] A. Pazy, Semigroups of Linear Operators and Applications to Partial Differential Equations, Applied Mathematical Sciences, Vol.44, Springer-Verlag, New York, 1983. MR710486 (85g:47061)

[24] M.Sermange and R. Temam, Some mathematical questions related to the MHD equations, Comm. Pure Appl. Math., 36 (1983), no. 5, 635-664. MR716200 (85k:76042)

[25] M.E. Taylor, Partial Differential Equations, Springer-Verlag, New York, 1996. MR:1395147 (98b:35002a)

[26] M.E. Taylor, Incompressible fluid flows on rough domains, pp. 320-334 in Semigroups of Operators: Theory and Applications, Progr. Nonlinear Differential Equations Appl., Vol.42, Birkhäuser, Basel, 2000. MR1788895 (2001i:35239)

[27] N. Yamaguchi, $L^{q}-L^{r}$ estimates of solution to the parabolic Maxwell equations and their application to the magnetohydrodynamic equations, in Proceedings of the 28th Sapporo Symposium on Partial Differential Equations, 2003.

Department of Mathematics, University of Missouri-Columbia, Columbia, Missouri 65211

E-mail address: marius@math.missouri.edu

LatP - UmR 6632, Faculté des Sciences de Saint-Jérôme - Case Cour A, Université Aix-Marseille 3, F-13397 Marseille Cédex 20, France

E-mail address: sylvie.monniaux@univ.u-3mrs.fr 